\title{
Modelling spectral and timing properties of accreting black holes: the hybrid hot flow paradigm
}

\author{
Juri Poutanen • Alexandra Veledina
}

Received: date / Accepted: date

\begin{abstract}
The general picture that emerged by the end of 1990s from a large set of optical and X-ray, spectral and timing data was that the X-rays are produced in the innermost hot part of the accretion flow, while the optical/infrared (OIR) emission is mainly produced by the irradiated outer thin accretion disc. Recent multiwavelength observations of Galactic black hole transients show that the situation is not so simple. Fast variability in the OIR band, OIR excesses above the thermal emission and a complicated interplay between the X-ray and the OIR light curves imply that the OIR emitting region is much more compact. One of the popular hypotheses is that the jet contributes to the OIR emission and even is responsible for the bulk of the X-rays. However, this scenario is largely ad hoc and is in contradiction with many previously established facts. Alternatively, the hot accretion flow, known to be consistent with the X-ray spectral and timing data, is also a viable candidate to produce the OIR radiation. The hot-flow scenario naturally explains the power-law like OIR spectra, fast OIR variability and its complex relation to the X-rays if the hot flow contains non-thermal electrons (even in energetically negligible quantities), which are required by the presence of the $\mathrm{MeV}$ tail in $\mathrm{Cyg} \mathrm{X}-1$. The presence of non-thermal electrons also lowers the equilibrium electron temperature in the hot flow model to $\lesssim 100 \mathrm{keV}$, making it more consistent with observations. Here we argue that any viable model should simultaneously explain a large set of spectral and timing data and show that the hybrid (thermal/non-thermal) hot flow model satisfies most of the constraints.
\end{abstract}

Keywords Accretion, accretion discs - black hole physics - radiation mechanisms: non-thermal $\cdot$ X-rays: binaries

PACS 04.25.dg $\cdot 52.25 . \mathrm{Os} \cdot 52.40 . \mathrm{Db} \cdot 95.30 . \mathrm{Jx} \cdot 97.80 . \mathrm{Jp} \cdot 98.62 . \mathrm{Mw}$

J. Poutanen, A. Veledina

Astronomy Division, Department of Physics, PO Box 3000, FI-90014 University of Oulu, Finland

E-mail: juri.poutanen@gmail.com, alexandra.veledina@oulu.fi 


\section{Introduction}

Models for accretion onto a black hole (BH) have been discussed now for more than 40 years. During the last 10-15 years we have seen a dramatic increase in the amount of information on the BH X-ray binaries (BHBs). Spectral details (iron lines and Compton reflection), spectral transitions, and variability on various time scales has been studied in unprecedented details with the new generation X-ray telescopes such as Rossi X-ray Timing Explorer (RXTE) and XMM-Newton. Excellent recent reviews are devoted to these advances (Zdziarski and Gierliński 2004; Remillard and McClintock 2006; Done et al. 2007; Done 2010; Gilfanov 2010).

In addition to the X-ray data, we have seen an explosion of information coming from other wavelengths: radio, sub-mm, infrared, optical, $\mathrm{UV}, \mathrm{MeV}$ and nowadays even from the $\mathrm{GeV}$ range. What is even more spectacular is that the properties of the $\mathrm{BHs}$ at these other wavebands are correlated with the X-ray flux and X-ray states. Among the most impressive achievements we find the discovery of correlated fast variability in the optical/infrared (OIR) band and in the X-rays Kanbach et al. 2001; Durant et al. 2008; Gandhi et al. 2008) with some hints actually coming already 30 years ago (Motch et al. 1983). This got theoreticians to scratch their heads and invent new models that often were in disagreement with previously established theories and contradicted many other available data.

Here we discuss some of the recent discoveries. We would like to note that the time for theoretical (phenomenological) models based purely on spectral properties are long gone. In order to be considered seriously, any model has to address many observed facts together.

This review consists of two parts. In the first one, we discuss the most recent reincarnation of the hot flow model, which now also considers the role of the nonthermal particles. In the second part, we discuss recent observational advances. We review the spectral data in various energy bands concentrating on the X-rays and the OIR. Then we discuss the observed temporal properties and correlated variability in different energy bands, as well as more complicated temporal-spectral statistics such as Fourier resolved spectra. In this review we will concentrate on the hard state and interpret the observations in terms of the hot flow model.

\section{Hot flow models}

\subsection{Comptonization models for the X-ray emission}

At high accretion rates exceeding typically $10 \%$ of the Eddington value, BHs are in the "soft state" and have thermally looking spectra peaking in the standard 2-10 keV Xray band, which are consistent with the thin $\alpha$-disc model (Shakura and Sunvaev 1973; Novikov and Thorne 1973). These thermally-dominated spectra presumably depend only on the accretion rate, the $\mathrm{BH}$ mass and spin and the inclination. They potentially can be used to determine the $\mathrm{BH}$ spin if the distance to the source and e.g. the $\mathrm{BH}$ mass and inclination are known (see McClintock et al. 2013). However, often strong powerlaw tails are seen (see Fig. 2). This tails are interpreted as a signature of non-thermal "corona" atop of the standard Shakura-Sunyaev disc (Fig. 10).

At the lower accretion rate, BHs are often found in the "hard state" and their spectra do not even remotely look thermal, but are close to the power-law in the X-ray band 


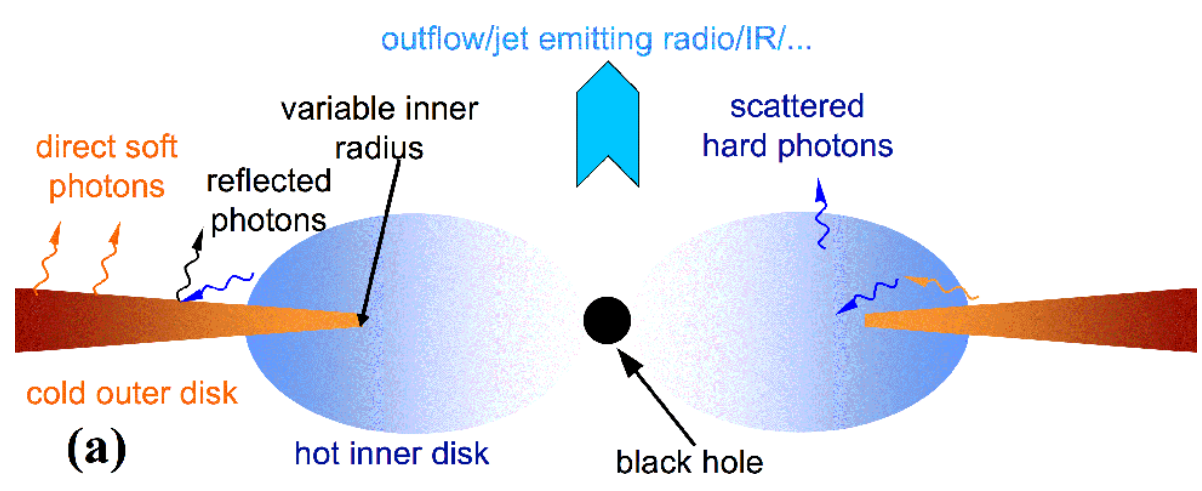

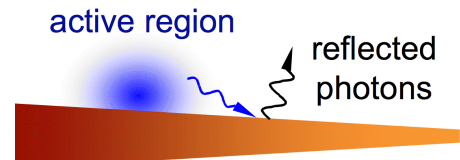

(b)

cold accretion disk

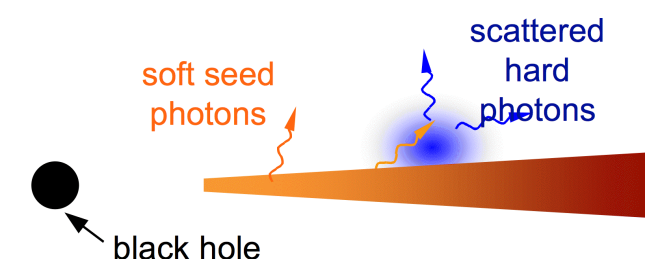

Fig. 1 (a) A schematic representation of the likely geometry in the hard state, consisting of a hot inner accretion flow surrounded by optically-thick cold accretion disc. The hot flow constitutes the base of the jet (with the counter-jet omitted from the figure for clarity). The disc is truncated far away from the minimum stable orbit, but it may overlap with the hot flow. The soft photons emitted by the disc (and possibly internally produced synchrotron photons) are Compton upscattered in the hot flow, and emission from the hot flow is partly Comptonreflected from the disc. (b) The likely geometry in the soft state consisting of flares/active regions above an optically-thick accretion disc extending close to the minimum stable orbit. The soft photons emitted by the disc are Compton upscattered in the flares by non-thermal electrons producing power-law spectra extending to $\gamma$-rays. Emission from the flares is partly Compton-reflected from the disc. From Zdziarski and Gierliński (2004).

with a sharp cutoff at about $100 \mathrm{keV}$ (e.g. Gierlinski et al. 1997; Zdziarski et al. 1998). These are well described by the thermal Comptonization model (see Zdziarski et al. 1997; Poutanen 1998, for reviews). The nature of the hard state emission and origin of the hot electrons has been already discussed in the 1970s (Shapiro et al. 1976; Ichimaru 1977) and is commonly associated with either a hot inner flow close to the BH (Esin et al. 1997, 1998; Poutanen et al. 1997; Naravan et al. 1998; Yuan and Zdziarski 2004) or a corona above the accretion disc (Galeev et al. 1979; Haardt and Maraschi 1993; Haardt et al. 1994; Stern et al. 1995b; Poutanen and Svensson 1996; Beloborodov 1999b, see Fig. 1h for a possible geometry).

Knowing the slope of the hard state spectra (with photon index $\Gamma=1.6-1.8$ ) we can easily estimate the ratio of the total emitted power $L$ to the soft seed photon luminosity $L_{\mathrm{S}}$ entering Comptonization region, i.e. the amplification factor $A=L / L_{\mathrm{S}}$. Beloborodov (1999a) found an approximate relation between $\Gamma$ and $A$ for the Comptonized spectra:

$$
\Gamma=\frac{7}{3}(A-1)^{-\delta},
$$

where $\delta=1 / 6$ for BHBs and $\delta=1 / 10$ for AGNs and the typical seed photon temperatures of $0.2 \mathrm{keV}$ for BHBs and $5 \mathrm{eV}$ for AGNs were assumed. If indeed the disc 
photons are being Comptonized, we get $A \approx 10$ for BHBs in their hard state. This fact puts serious constraints on the geometry of the emission region Poutanen 1998; Beloborodov 1999a) and immediately rules out simple slab-corona models which predict much smaller amplification $A \lesssim 2$ and softer spectra because of the efficient X-ray reprocessing in the cold disc (Stern et al. 1995b). Assuming that coronal plasma has a mildly relativistic velocity away from the cold disc Beloborodov 1999b; Malzac et al. 2001) one can in principle reconcile that model with the observed slopes as well as with the correlated changes of the spectral hardness and the amount of Compton reflection from the disc, but still one would have troubles explaining their correlations with the iron line width and characteristic variability frequencies (see Sect. 3.1).

If the accretion flow geometry is such that the inner part is occupied by the hot flow and the outer is the standard cold disc, the seed photons for Comptonization might be internal to the hot flow or come from the outer disc. For the truncation radius of the cold disc of more than $30 R_{\mathrm{S}}$ (where $R_{\mathrm{S}}=2 G M / c^{2}$ is the Schwarzschild radius), most of the disc photons go directly to the observer and therefore the disc should be very prominent in the total spectrum. Furthermore, the luminosity in disc photons being Comptonized in the region of major gravitational energy release $\left(<10 R_{\mathrm{S}}\right)$ would be only about $1 \%$ of the total luminosity resulting in an amplification factor of a hundred and a very hard Comptonization spectrum (see equation 11). Neither is observed. An overlap of the inner hot flow with the cold disc (Poutanen et al. 1997; see Fig. 19) was proposed as a solution to this, but does not really solve the problem, because most of the energy is dissipated within $10 R_{\mathrm{S}}$. Thus the disc should come to radii below $10 R_{\mathrm{S}}$. Another solution is that cold clouds embedded into the flow reprocess hard photons (Celotti et al. 1992; Krolik 1998; Zdziarski et al. 1998; Poutanen 1998) increasing thus the number of seed soft photons.

Alternatively, the hot flow itself can generate enough soft photons by synchrotron emission of the same hot electrons that emit the X-rays, if the electron temperature $T_{\mathrm{e}}$ is sufficiently high to overcome the self-absorption problem. The spectrum from the hot optically thin advection-dominated accretion flows (ADAF) indeed is produced mostly by synchrotron self-Compton (SSC) mechanism Narayan and Yi 1994, 1995; Naravan et al. 1998; Yuan and Narayan 2014). Detailed radiative transfer calculations accounting for non-local Compton effect coupled with dynamics (also in the Kerr metric) predict, however, $T_{\mathrm{e}}$ exceeding the observed values of $50-100 \mathrm{keV}$ by at least a factor of 2 (see fig. 3 in Yuan et al. 2007, fig. 1d in Xie et al. 2010 and figs. 5 and 6 in Niedźwiecki et al. 2012). Most of the problems get solved if the electrons have a reasonably strong non-thermal tail. In this case, synchrotron emission becomes much more efficient (Wardziński and Zdziarski 2001) increasing the cooling, softening the spectrum and lowering $T_{\mathrm{e}}$ to the values which agree with observations. This is the essence of the hybrid Comptonization (or rather hybrid SSC) models developed for bright accreting BHs (Poutanen and Coppi 1998; Coppi 1999; Poutanen and Vurm 2009; Malzac and Belmont 2009; Veledina et al. 2011b, 2013a) that are described below in more details. Non-thermal electrons can also play a role in low-luminosity systems such as Sgr A* (Mahadevan 1998; Özel et al. 2000; Yuan et al. 2003). However, in these conditions the equilibrium electron temperature is very high $(\sim \mathrm{MeV})$ and the optical depth is very low, so that thermal synchrotron radiation is very effective. The role of the non-thermal electrons is then reduced to production of tails at higher and lower frequencies around the dominating thermal synchrotron peak. 

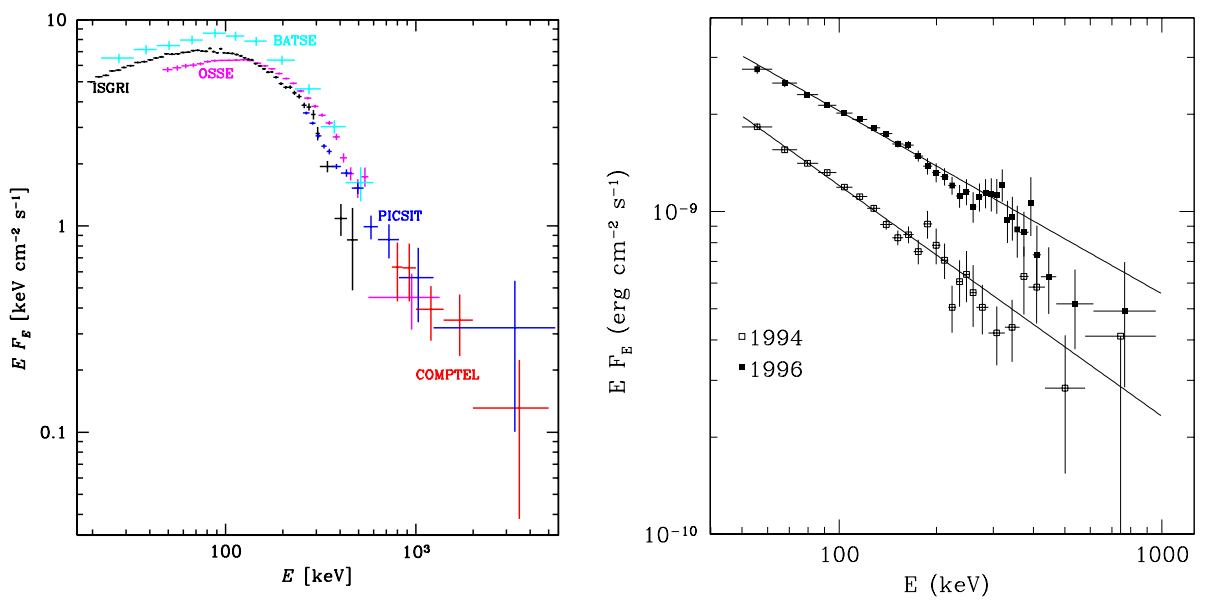

Fig. 2 Hard (left panel) and soft (right) state spectra of Cyg X-1. From Zdziarski et al. (2012) and Gierliński et al. (1999).

\subsection{Hybrid Comptonization model}

Arguments in favour of the presence of non-thermal particles in the accretion flow come from the significant detection of the MeV tails in the hard state spectra of Cyg X-1 (McConnell et al. 1994, 2002; Ling et al. 1997; Jourdain et al. 2012a; Zdziarski et al. 2012, see Fig. 2) and marginally in GX 339-4 (Droulans et al. 2010). Also in the soft state, power-law tails extending to hundreds of $\mathrm{keV}$ and up to possibly $10 \mathrm{MeV}$ are present (Grove et al. 1998; Gierliński et al. 1999; Zdziarski et al. 2001; McConnell et al. 2002, see Fig. 2) and are well described by non-thermal/hybrid Comptonization (Poutanen 1998; Poutanen and Coppi 1998; Gierliński et al. 1999; Coppi 1999). What is the nature of the non-thermal particles is an open question.

On the theoretical ground, it is expected that the electrons get some of the energy via Coulomb collisions with hot, nearly virial protons (as assumed in ADAF models) or diffusive acceleration by MHD turbulence, resulting in "thermal heating", i.e. energy is transferred to the thermal population of electrons. However, some fraction of the energy can be transferred to them in the form of injection of relativistic electrons by shock acceleration (Fragile and Blaes 2008; Das et al. 2009; Henisev et al. 2012), magnetic reconnection (Ding et al. 2010; Riquelme et al. 2012; Hoshino 2013), or electron-position pair production by decay of pions born in proton-proton collisions (Mahadevan 1998). Because the microphysics of electron acceleration and heating in the hot flow is not well established from first principles, one can use a phenomenological prescription, where some fraction of the total power is given to the electrons as heating and the rest of the energy is given by non-thermal injection of power-law electrons. Such hybrid thermal/non-thermal models are reviewed by Coppi (1999). Models with the least number of free parameters are either purely thermal or purely non-thermal. Because the first option clearly contradicts the data, we consider in the following the second non-thermal option. Of course, the assumption that the electrons receive $100 \%$ of their energy in the form of non-thermal injection is not realistic. Fortunately, the results are not very sensitive to the actual value of non-thermal injection fraction as long as it exceeds 10\% (see Appendix B1 in Veledina et al. 2013a). The reason is that 
even for pure non-thermal injection the steady-state electron distribution is thermal at low energies due to thermalisation via Coulomb collisions between electrons as well as via synchrotron self-absorption Ghisellini et al. 1988, 1998; Nayakshin and Melia 1998; Vurm and Poutanen 2009). At higher energies a tail develops, whose shape is determined by the injection and the competition between various cooling/thermalisation mechanisms.

The most advanced hybrid models solve simultaneously for the momentum distribution of all considered particles, electrons and positrons (sometimes also protons), as well as the photons. This either can be done via Monte-Carlo simulations (e.g. Stern et al. 1995a), or by solving coupled kinetic equations (Coppi 1992, 1999; Belmont et al. 2008; Vurm and Poutanen 2009). The processes that need to be accounted for under the conditions of the hot flows are Compton scattering, synchrotron emission and absorption, pair production, Coulomb collisions (between leptons as well as with protons), and bremsstrahlung. The radiative transfer can be easily handled exactly with Monte-Carlo approach, while usually with the kinetic approach an escape probability formalism in a single-zone approximation is used. A single-zone approximation works reasonably well if most of the escaping radiation at all wavelengths is dominated by some narrow range of radii. For the X-ray production this is fine, because most of the energy is dissipated in the accretion flow spread from say $3 R_{\mathrm{S}}$ to $10 R_{\mathrm{S}}$. On the other hand, this approximation fails in the OIR. Here the outer zones of the hot flow can dominate the energy output in those bands as the inner zones are opaque for that radiation. Because the radiation from the inner zones can affect the energy balance and the escaping radiation from the outer zones, a multi-zone treatment with the radiative transfer is required (Veledina et al. 2013a).

The simplest model is described by the size, the magnetic field strength $B$, Thomson optical depth $\tau$, the total injected power $L$, the spectrum of the injected electrons and the spectrum and luminosity of the external (blackbody/cold accretion disc) photons. For an extended multi-zone flow one can assume that the electron energy injection rate as well as $B$ and $\tau$ have power-law distributions with radius, $B(R) \propto R^{-\beta}, \tau(R) \propto$ $R^{-\theta}$ and thus the additional parameters, e.g., $\beta$ and $\theta$ have to be introduced. Many parameters can be directly determined from observations or taken from theoretical accretion disc models. Now let us describe the main properties of such hybrid models.

\subsection{Basic properties of hybrid accretion flows}

If the truncation radius of the cold disc is significantly larger than the region of the major energy dissipation (i.e. $R_{\mathrm{tr}}>30 R_{\mathrm{S}}$ ), then the X-ray spectrum is dominated by the radiation from the innermost zone of the hot flow. Here locally generated nonthermal synchrotron photons are Comptonized by the thermal electron population (see Fig. 3a). Here we note that the internally generated synchrotron photons are much more efficient in cooling the plasma than the external disc photons. The first obvious difference comes from the geometry: all synchrotron photons are injected within the hot flow and have a chance to be Comptonized, while in the disc case only a small fraction gets to the hot flow. The second difference comes from the fact that the synchrotron photons have much smaller energies than the cold disc photons. Therefore, in order to produce the same spectral slope of the Comptonization continuum (with nearly the same total power), the synchrotron luminosity can be smaller by a factor a several than 

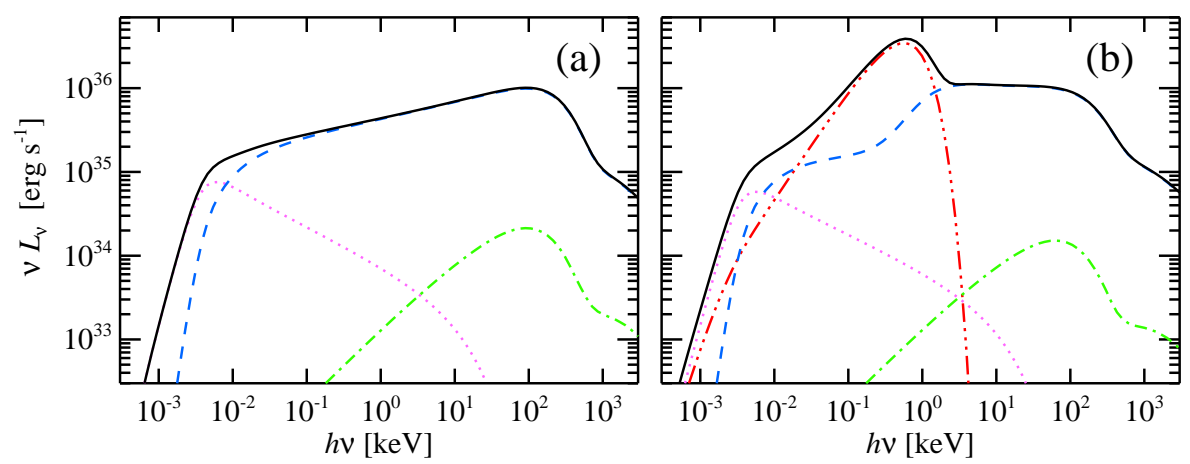

Fig. 3 Spectrum from the one-zone hot flow. (a) Spectral decomposition of the hybrid SSC model. The pink dotted line is the non-thermal synchrotron, the blue dashed is its Comptonization spectrum, and the green dot-dashed curve is the bremsstrahlung component. (b) Same as (a), but with the disc photons (red triple-dot-dashed curve) dominating the seed photons distribution. Now below $1 \mathrm{keV}$ Comptonization spectrum is produced by SSC, while at higher energies Comptonization of the cold disc photons dominates.

the disc luminosity intercepted by the hot flow. In other words, the amplification factor given by equation (1) is now closer to 50 than 10 (because we need to use $\delta \approx 1 / 10$ ).

At a few per cent of the Eddington luminosity corresponding to the bright hard state, the low-energy electrons are thermalised by Coulomb collisions and synchrotron self-absorption to the typical electron temperatures $T_{\mathrm{e}}$ of about $100 \mathrm{keV}$ (Poutanen and Vurm 2009; Malzac and Belmont 2009; Veledina et al. 2011b). The hybrid model produces surprisingly stable spectra with photon index $\Gamma \sim 1.6-1.8$ largely independent of the model parameters (Fig. 37). The high-energy electron tail can be approximated by a power-law, which is softer than the injected distribution due to the cooling. The observed $\mathrm{MeV}$ tail is produced by Compton up scattering of the $100 \mathrm{keV}$ photons by these non-thermal electrons. The outer zones of the hot flow have softer spectra because of the additional cooling by the cold disc photons and because of more transparent conditions for the synchrotron radiation (see Fig. 3b). The overall X-ray spectrum is thus concave.

The OIR spectrum consists of two components: the multi-colour (possibly irradiated) cold accretion disc and the synchrotron radiation from the hot flow. Similarly to the inhomogeneous synchrotron models developed for extragalactic jets Marscher 1977; Blandford and König] 1979), the non-thermal synchrotron spectrum of the hot flow is a power-law $F_{\nu} \propto \nu^{\alpha}$ with the index (Veledina et al. 2013a):

$$
\alpha_{\mathrm{OIR}}=\frac{5 \theta+\beta(2 p+3)-2 p-8}{\beta(p+2)+2 \theta},
$$

where $p$ and $\theta$ are the indices of the equilibrium distribution of electrons, $n_{\mathrm{e}}(R, \gamma) \propto$ $R^{-\theta} \gamma^{-p}$, at Lorentz factor $\gamma$ emitting at the self-absorption frequency. Typically, spectral indices are $\alpha_{\mathrm{OIR}} \sim 0 \pm 0.5$. The turn-over at longer wavelengths is determined by the extent of the hot flow, while the transition to the optically thin synchrotron emission is hidden by the Comptonization spectrum (Fig. 33).

The disc spectrum can be split also into two components: the inner warmer standard disc heated by viscous forces and the outer cooler disc heated by the X-rays. In the OIR one expects the dominance of the irradiated disc, which has the radial temperature dependence $T_{\text {irr }} \propto R^{-3 / 7}$ (Cunningham 1976). Presence of the irradiated disc can 

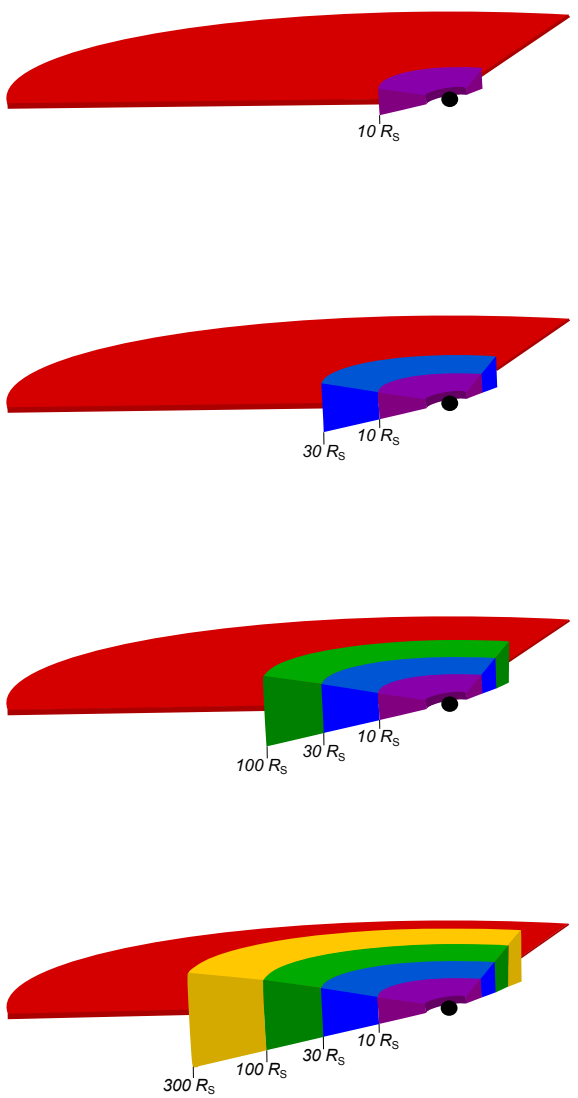
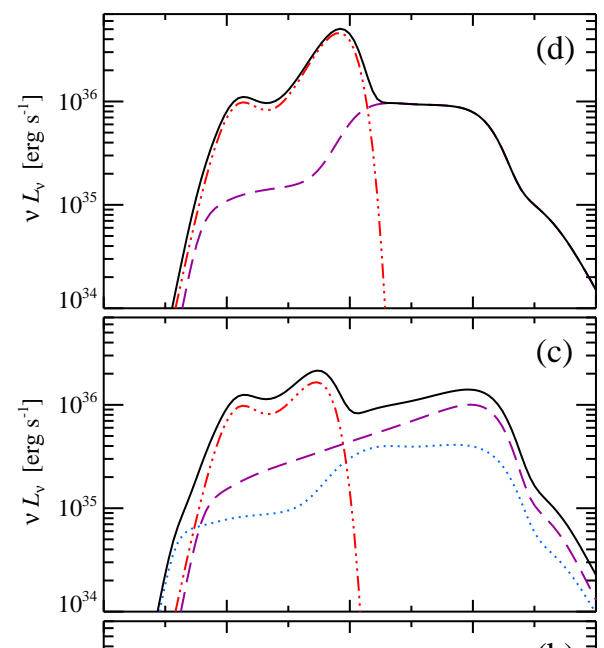

(b)

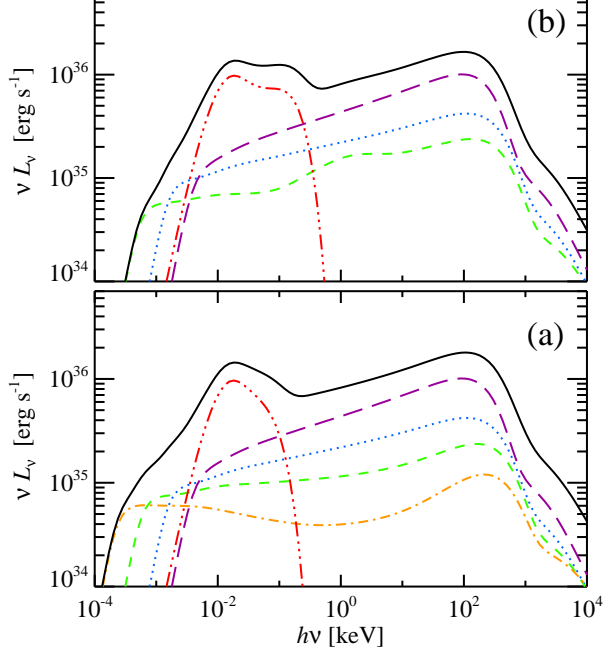

Fig. 4 Left panels: Schematic picture of the evolution of the hot flow size during spectral state transitions. In the low-luminosity hard state, the geometrically thick inner hot flow is large dominating the radiative energy output. With increasing accretion rate (from lower to upper panels) the outer zones of the hot flow gradually collapse swallowed by the cold thin accretion disc. For illustrative purposes, the hot flow is split into four zones with outer radii $10 R_{\mathrm{S}}$ (violet, zone 1 ), $30 R_{\mathrm{S}}$ (blue, zone 2), $100 R_{\mathrm{S}}$ (green, zone 3 ) and $300 R_{\mathrm{S}}$ (yellow, zone 4 ). Red outer component represents the truncated cold accretion disc. Right panels: corresponding spectral evolution at the state transition. Contribution of different zones are marked with different lines: zone 1 (violet long-dashed), zone 2 (blue dotted), zone 3 (green short-dashed), zone 4 (orange dot-dashed) and outer cold irradiated disc (red three-dot-dashed). At the lower panel, the hot flow spectrum was calculated not accounting for the seed photons from the disc. An IR excess is clearly visible above the irradiated disc spectrum. Collapse of the hot flow leads to dramatic changes in the OIR hot flow synchrotron spectrum. Changes in the X-ray spectral shape are insignificant until the truncation radius becomes as small as $10 R_{\mathrm{S}}$. The Comptonization spectrum from the hot-flow zone closest to the cold disc consists of two separate continua produced by Comptonization of the synchrotron and the cold disc photons, with the latter being dominant source of seed photons in this zone (see also Fig. 3b). For simplicity, in this illustration the total luminosity is kept constant. Adapted from Veledina et al. (2013a). 
be reflected in the optical echoes (Hynes et al. 1998; O'Brien et al. 2002), the X-ray time-lags (see Poutanen 2002, and references therein) and in the optical/X-ray crosscorrelation function (Hynes et al. 2009a; Veledina et al.|2011a). Its signatures are also seen in the spectrum (e.g., Hynes et al.|2002; Gierliński et al.|2009). For typical parameters of LMXBs with the disc size of $10^{11} \mathrm{~cm}$ and the X-ray luminosity of $10^{37} \mathrm{erg} \mathrm{s}^{-1}$ the temperature of the outer disc is about $T_{\mathrm{irr}} \sim 20000 \mathrm{~K}$. The relative role of the components varies with the wavelength. The disc spectrum is hard in the OIR band, while the hot flow produces an excess emission dominating below $\sim 1 \mathrm{eV}$ (see Fig. 4a and Fig. 6).

At smaller accretion rates below a few percent of the Eddington value, the flow becomes more transparent to the synchrotron photons leading to their increasing role in cooling and resulting in slightly softer X-ray spectra (Veledina et al. 2011b). At higher accretion rates associated with the transition to the soft state (see Fig. 4), the outer zones of the hot flow gradually collapse, so that the disc truncation radius $R_{\text {tr }}$ decreases (Poutanen et al. 1997; Esin et al. 1997). This leads to the rising role of the disc as a source of seed photons, which increases Compton cooling, leads to spectral softening and causes changes in the electron distribution from mostly thermal to nearly nonthermal (Poutanen and Coppi 1998; Poutanen and Vurm 2009; Malzac and Belmont 2009; Veledina et al. 2011b). This transition is accompanied by the increase in the reflection amplitude that scales with the solid angle at which the cold disc is seen from the hot flow. The OIR hot flow luminosity drops first at longer wavelengths, where the outer zones radiate (see Fig. 4). Note that the X-ray spectrum changes much later, when the truncation radius comes closer to the zone of the main energy dissipation of about $10 R_{\mathrm{S}}$. At this moment, the Comptonization spectrum of the remaining hot flow consists of two segments: the hybrid SSC dominates at lower energies, while Comptonization of the disc photons takes over at energies above the cold disc peak (see Fig. 3b). Similarly curved spectra are expected for the hot flow zones closest to the cold disc (Fig. (4). The corresponding time delay between sharp luminosity changes at different wavelengths scale with the timescale of state transition and, depending on the separation of the wavelengths and accretion parameters, can be as short as hours (e.g., if one observes in different optical filters), as long as a few days (e.g., IR and UV) or weeks (e.g. IR and X-rays). The opposite evolution should be observed in the soft-to-hard spectral transition when the accretion rate drops after the outburst peak. Here first the X-ray spectral transition starts and at a timescale of a week or so the emission in the OIR peaks, when the size of the hot flow becomes large enough for the OIR synchrotron photons to escape.

At a high accretion rate, the accretion disc extends to the last stable orbit and the source switches to the soft state. In this case, no inner hot flow exists, but a non-thermal magnetically powered corona still could be present (Fig. 1b). Its presence is supported by the existence of the X-ray $/ \gamma$-ray power-law tails. The non-thermal synchrotron from the corona may be present in the OIR band, but at a much lower level, because the electron cooling is dominated now by Comptonization of the disc photons. Actually, such a non-thermal corona atop the cold disc (in addition to the hot inner flow) may be present also in the hard state, but its emission scaled with the cold disc luminosity is weak. 

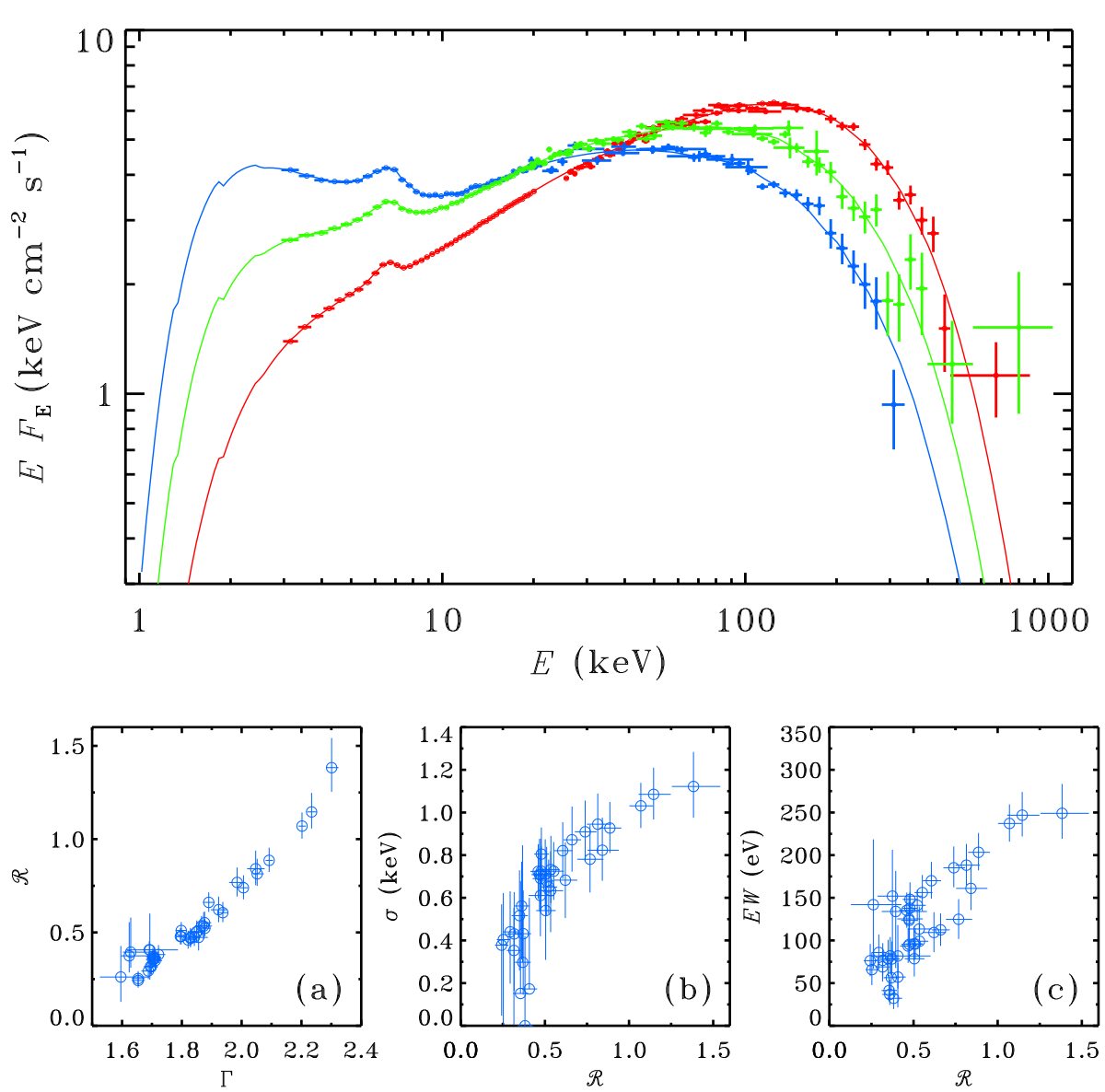

Fig. 5 Spectral variations of Cyg X-1 in its hard/intermediate state as shown in the top panel. The lower panels show correlation between the amplitude of Compton reflection $\mathcal{R}$, photon spectral index $\Gamma$, width $\sigma$ and the equivalent width of the iron line. Adapted from Ibragimov et al. (2005).

\section{Observational properties}

\section{$3.1 \mathrm{X}$-ray $/ \gamma$-ray spectra}

Let us first briefly summarise what we know about the spectral properties of BHBs. Further details can be found in reviews by Poutanen (1998), Zdziarski and Gierliński (2004), Done et al. (2007), and Done (2010). In the hard-state, the spectra constitute a power-law in the X-ray band with a rather stable spectral slope (with photon index $\Gamma \sim 1.6-1.9$ and ubiquitous sharp cut-off at around $100 \mathrm{keV}$ (Gierlinski et al. 1997; Zdziarski et al. 1998; Ibragimov et al. 2005; see fig. 10a in Zdziarski and Gierliński 2004). The shape of the spectra allows us to conclude that they are produced by (nearly) thermal Comptonization (e.g. Poutanen 1998; Zdziarski and Gierliński 2004, see Fig. 5). When the high-quality data above $100 \mathrm{keV}$ were available (e.g. with OSSE/CGRO or IBIS/INTEGRAL or HXD-GSO/Suzaku) the electron temperature 
(measured with the accuracy of about 10\%) 1 was always lying in the interval 50-120 keV (e.g. Gierlinski et al. 1997; Zdziarski et al. 1998; Poutanen 1998; Aref'ev et al. 2004; Makishima et al. 2008), with temperature increasing with decreasing luminosity. The hard-state accreting BHBs also show weak but distinctive MeV tails (McConnell et al. 1994, 2002; Ling et al. 1997; Droulans et al. 2010; Jourdain et al. 2012a; Zdziarski et al. 2012, see Fig. 2). Such spectra are consistent with the hybrid hot-flow model. During the transition to the soft state, $T_{\mathrm{e}}$ is reduced. However, because of the growing importance of non-thermal tail, the spectral cutoff energy may actually increase (Poutanen and Vurm 2009).

The fact that the spectra are stable in the hard state with variable luminosity and never have $\Gamma<1.6$ argues in favour of the hybrid SSC as the main emission mechanism. If the outer cold disc were the seed photons provider, one expects harder and strongly variable spectra when the truncation radius is changing in the soft-to-hard transition. Moreover, the best studied BHs, Cyg X-1 and GX 339-4, clearly have a concave spectrum that can be fitted with two Comptonization continua (Frontera et al. 2001; Ibragimov et al. 2005; Makishima et al. 2008; Shidatsu et al. 2011; Yamada et al. 2013). The inhomogeneous hot flow model naturally explains such spectra by the radial dependence of the slope of Comptonization spectrum. The spectral curvature can also appear if a non-thermal corona (similar to that producing power-law tail in the soft state) exists above the cold disc during the hard state too (Ibragimov et al. 2005).

In addition to the smooth continuum, in both states a Compton reflection feature and the fluorescent iron line at $6.4 \mathrm{keV}$ originating from cool opaque matter (likely the cool accretion disc) are often detected. The strength of Compton reflection is correlated with the X-ray slope (Zdziarski et al. 1999, 2003), with the width of the iron line as well as with the quasi-periodic oscillation (QPO) frequency (Gilfanov et al. 1999; Revnivtsev et al. 2001; Ibragimov et al. 2005; Gilfanov 2010, see lower panels in Fig. 50. During the outbursts of $\mathrm{BH}$ transients, in the hard state the iron line width correlates well with the luminosity (Kolehmainen et al. 2013). These data are consistent with the hot-flow paradigm where all correlation are basically controlled by the cold disc truncation radius.

At luminosities above a few per cent of Eddington, BHBs show a strong correlation between spectral index and luminosity. At lower luminosities the trend is reversed, i.e. spectra become softer with decreasing luminosity $\mathrm{Wu}$ and $\mathrm{Gu}$ 2008; Sobolewska et al. 2011). Similarly, an indication of the reverse trend was detected in low-luminosity AGNs (Constantin et al. 2009; Gu and Cao 2009). This was interpreted as a change of the source of seed photons for Comptonization from the disc photons dominating at higher luminosities to the synchrotron at lower luminosities. The whole spectral index - luminosity dependence is well explained by the hybrid hot flow model (see figs 7 and 12 in Veledina et al. 2011b). At very small luminosities, the flow becomes more transparent for the synchrotron radiation increasing the photon input and softening the Comptonization spectra.

\footnotetext{
1 Note that the electron temperature can be measured only if high-quality data are available above $100 \mathrm{keV}$ and accurate Comptonization models such as COMPPS Poutanen and Svensson 1996) or EQPAIR (Coppi 1999) are used for fitting. Using the exponentially cut-off power-law for the fits and identifying the e-folding energy with the electron temperature is dangerous, because that model does not correctly describe the shape of Comptonization continuum (see e.g. fig. 5 in Zdziarski et al. 2003). This can result in over-estimation of $T_{\mathrm{e}}$ by a factor of 3-6.
} 


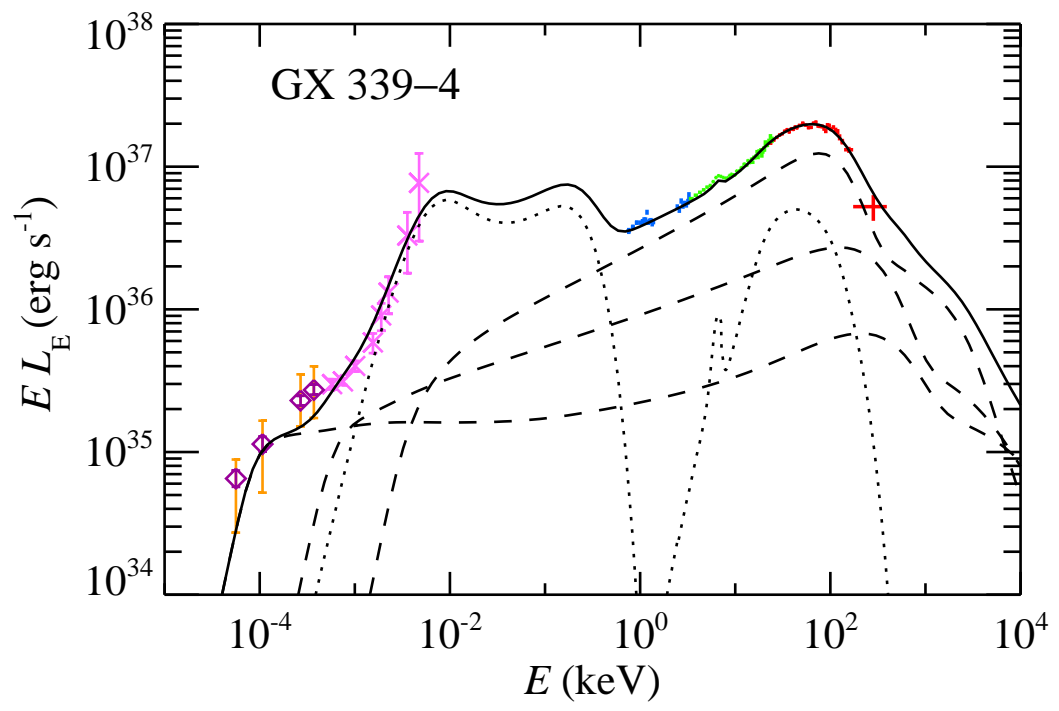

Fig. 6 Broad-band spectrum (corrected for absorption) of GX 339-4 in its hard state around March 5, 2010 from the mid-IR to the hard X-rays (Cadolle Bel et al. 2011). The dashed lines show contribution of different zones of the hot accretion flow (Veledina et al. 2013a), the dotted lines represent the spectra of the irradiated and standard discs as well as Compton reflection. From Veledina et al., in prep.

\subsection{Broad-band spectra and infrared flares}

Numerous multiwavelength campaigns were conducted over the past decade. Broadband radio to X-ray spectral energy distributions (SEDs) for many BHBs were constructed (e.g. Hynes et al. 2000; McClintock et al. 2001; Chaty et al. 2003; Cadolle Bel et al. 2007, 2011; Durant et al. 2009). The OIR emission is normally dominated by the (irradiated) disc, but the IR excesses are observed in a number of sources: XTE J1859+226 (Hvnes et al. 2002), GX 339-4 (Gandhi et al. 2011; Shidatsu et al. 2011; Buxton et al. 2012; Dincer et al. 2012; Rahoui et al. 2012), A0620-00 (Gallo et al. 2007), SWIFT J1753.5-0127 (Chiang et al. 2010), V404 Cyg (Hynes et al. 2009b), XTE J1550-564 (Jain et al. 2001; Russell et al. 2011). In some cases the OIR spectrum can be described by a pure power-law $F_{\nu} \propto \nu^{\alpha}$ with index $\alpha$ close to zero (e.g. $\alpha_{\mathrm{OIR}}=-0.15$ in XTE J1118+480, see Esin et al. 2001; Chaty et al. 2003). Sometimes the OIR excess spectrum is rather soft with $\alpha \approx-0.7$ (see Kalemci et al. 2013, for a recent overview). The IR excesses were previously explained by the jet (Hynes et al.|2002; Gallo et al. 2007) or the dust heated by the secondary star (Muno and Mauerhan 2006). Veledina et al. (2013a) recently argued that the OIR excess emission may also be produced by synchrotron radiation from the hot flow.

Here we only discuss a few representative examples of the recent studies. The broad-band spectrum of GX 339-4 in its hard state (Cadolle Bel et al. 2011, 2013) is shown in Fig. [6 The X-ray spectrum peaking at $\sim 100 \mathrm{keV}$ is well described by thermal Comptonization. The irradiated disc presumably dominates in the UV band. There is a clear excess in the mid- and near-IR (Gandhi et al. 2011), but the spectrum becomes harder at longer wavelengths. These data are well explained by the nonthermal synchrotron emission from the hot flow (Veledina et al. 2013a) of about $500 R_{\mathrm{S}}$. 

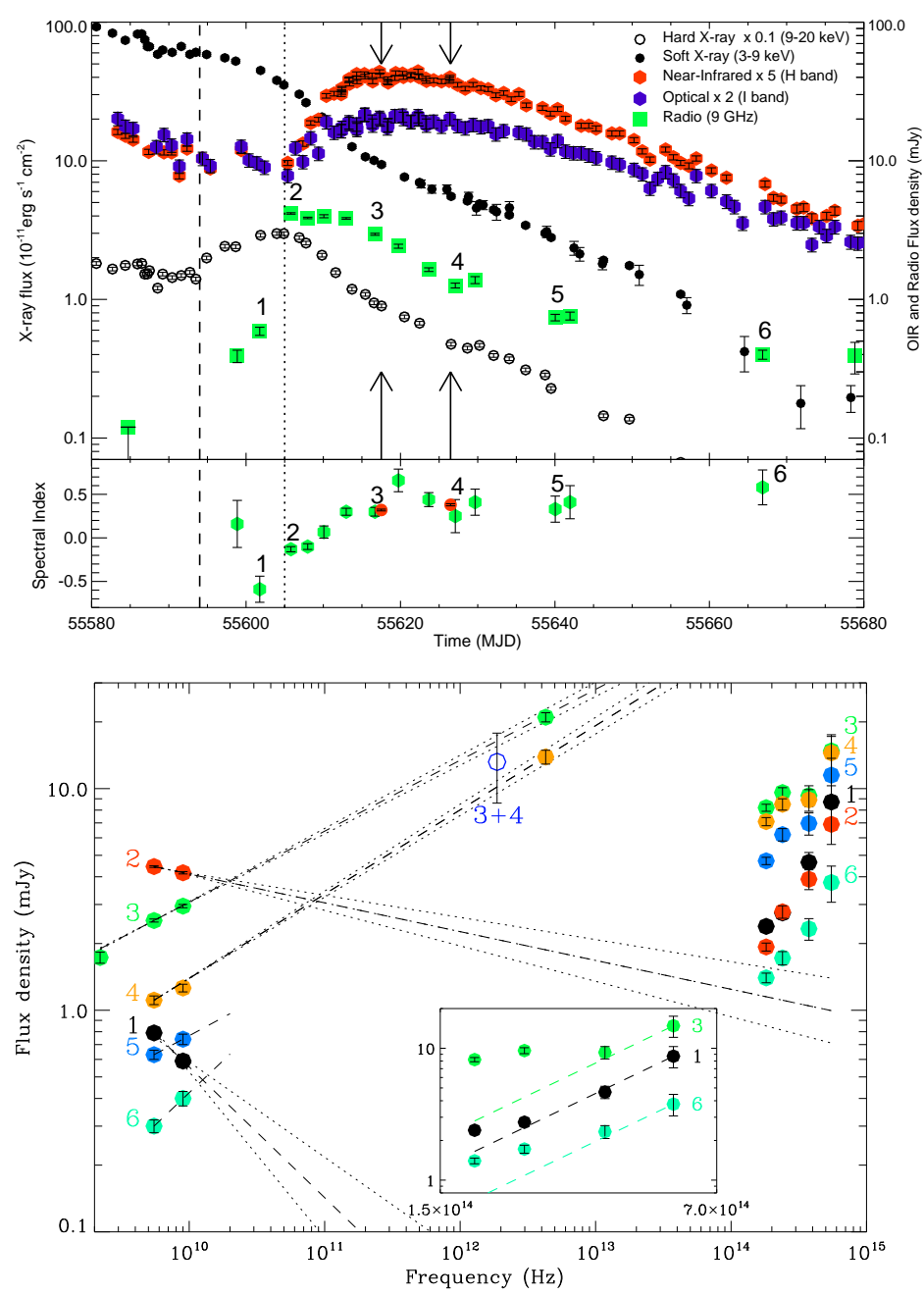

Fig. 7 Upper panel: The lightcurves of GX 339-4 during the decay of the 2010-2011 outburst in radio (green), NIR (red), optical (blue), soft X-ray (filled black circles) and hard X-ray (open black circles). The bottom sub-panel shows the evolution of the radio spectral index. The dashed vertical line indicates the start of the soft-to-hard state transition, while the dotted line marks the start of the OIR flare and the end of the transition (see fig. 1 in Dincer et al. 2012). The numbers refer to the individual observations displayed in the lower plot. Lower panel: Evolution of the radio to OIR spectra of GX 339-4. The inset zooms on the OIR spectra, with the dashed lines corresponding to power-law with $\alpha=1.5$ (for an irradiated disc). From Corbel et al. (2013).

Excellent data covering both radio and OIR bands have been collected during the 2010-2011 outburst of GX 339-4 Cadolle Bel et al. 2011; Dincer et al. 2012; Corbel et al. 2013, see Fig. 7). A week after the start of the transition to the hard state (marked by the vertical dashed line) the OIR spectrum has a clear soft excess above the reprocessing thermal emission (point 1), while the radio jet optically thin 
emission can contribute at most $1 \%$ to the OIR. Few days later, when the transition was completed (point 2), the fluxes in the $H$ and $I$-band show a sharp increase and an obvious IR excess in the OIR spectra is visible, while the radio emission is still soft with $\alpha \sim-0.1$. On a week time scale the radio spectrum transits to the harder, optically thick state with $\alpha \sim 0.5$ (point 3) corresponding to the synchrotron emission from an inhomogeneous source analogously to the extragalactic jets (Blandford and König] 1979). The radio spectrum stays hard and the IR excess is visible during the following decay. The high flux in the Herschel far-IR band lies exactly on the extrapolation of the radio spectra. Because after the break, the jet spectrum must be optically thin and soft, while the OIR spectra are flat (even after subtracting thermal component, as discussed by Dincer et al. 2012; Buxton et al. 2012; Corbel et al. 2013), the jet does not contribute significantly to the OIR bands. An important conclusion from these data is that there is a rather strong, evolving component in the OIR band which cannot be produced by the jet or reprocessing in the accretion disc at any stage of the outburst. Its appearance in the hard state is, however, consistent with the hot flow interpretation.

The IR excess similar to that seen in GX 339-4 in the hard state appears also in XTE J1550-564 (Jain et al. 2001), 4U 1543-47 (Buxton and Bailyn 2004) and XTE J1752-223 (Russell et al. 2012). The properties of the excess can be studied using the colour-magnitude diagram (see Fig. 8 r). During the 2000 outburst of XTE J1550-564 the data in the soft state, soft-to-hard transition and at very low-luminosity hard state can be adequately described by the reddened irradiated disc emission. Both at the rising (filled symbols) and decaying (open symbols) phase of the outburst, we see "flares" during which the spectrum becomes redder. One can interpret these flares as the appearance of the additional red component. Fitting the fluxes at the decaying stage with an exponential plus a constant, one can subtract the contribution from the irradiated disc and obtain a spectrum of the flare component only. We see that the second flare starts with the spectral index $\alpha \sim+0.7$, then becomes softer with $\alpha \sim-0.2$, and then harder again (see green arrows in Fig. 8). (The final points have large errors, because of the uncertainties in the subtraction of the disc.) What is important that the flare starts in $I$ before $H$, so that the index measured between filters $I$ and $H$ is even larger, $\alpha_{I H} \approx 1.0$. This behaviour rules out immediately the interpretation of the flare in terms of optically thin jet emission 2 Instead the data are consistent with the inhomogeneous hot accretion flow model of Veledina et al. (2013a). Such indices were also observed in the flare spectrum of XTE J1752-223 (Russell et al. 2012) and GX 339-4 (Dincer et al. 2012; Buxton et al. 2012). This would mean that all these OIR flares are produced by the hot flow but not the jet. The observed sharp colour change during the flares is related to the collapse/recover of a zone in the hot flow that is responsible for the H-band emission.

The delay of the IR flare peak by about 10 days from the start of the soft-to-hard transition is naturally expected, because the X-ray transition corresponds to the start of the retraction of the cold disc $\left(R_{\mathrm{tr}} \sim 10 R_{\mathrm{S}}\right)$, while the IR flare peaks when the hot

2 Colour-magnitude diagram for the 2000 outburst of XTE J1550-564 was constructed by Russell et al. (2011), who also related the observed colours to the intrinsic spectral indices and claimed very soft spectrum of the flare. Unfortunately, all their formulae are wrong for various reasons and the actual intrinsic spectra are much harder. Furthermore, the exponential fits to the OIR light curves to evaluate the flare spectrum were also flawed, as their fits overestimate the disc contribution in the $V$ and $I$ filters just before the flare (see fig. 2 in Russell et al. 2010) resulting in over-subtraction of the flux in those filters and in a much too soft spectrum of the flare (compare our $\alpha \sim+0.7$ at the start of the flare with their $\alpha \sim-1.6$ ). 

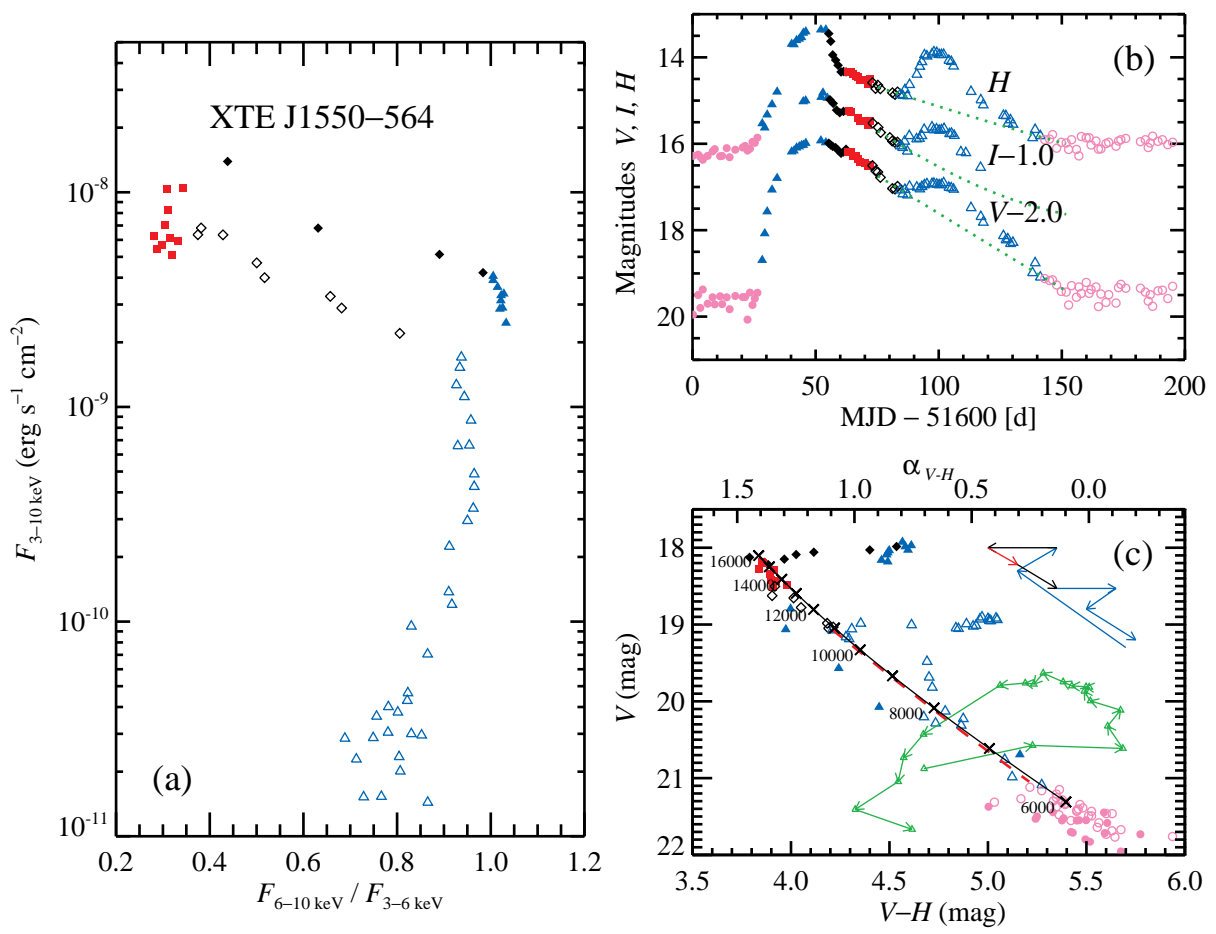

Fig. 8 (a) X-rays flux - hardness diagram for XTE J1550-564 during the 2000 outburst. Different colours indicate various stages of the outburst based on their X-ray hardness. The blue colour indicate the hard state, the black colour is the transition and the red colour the soft state. Open and closed symbols correspond to the rising and decaying outburst stages, respectively. (b) The light curves in $V, I$ and $H$-filters of XTE J1550-564 (Jain et al. 2001). The pink circles correspond to the quiescent state. The green dotted curves show the bestfit model for the decaying disc component. (c) The observed $V$ vs $V-H$ colour-magnitude diagram. The black solid line represent the relation expected for the black body disc of a characteristic radius $2.7 \times 10^{11} \mathrm{~cm}$ inclined at $i=75^{\circ}$ at distance of $4.38 \mathrm{kpc}$ (Orosz et al. 2011) of different temperatures (marked next to the line). The model magnitudes were reddened following the extinction law of Fitzpatrick (1999) with $A_{V}=5.0$. Much smaller $A_{V}$ (that would lead to softer spectrum) is not possible, because the disc temperature would be below hydrogen ionisation temperature needed for the outburst to start. The blue-black-red arrows illustrate schematically the time evolution of the source during the outburst. The green arrows show the path followed by the flare after MJD 51680 (see the text). The upper x-axes show the intrinsic spectral index $\alpha_{V H}=4.63-0.84(V-H)$ computed from the observed colour. We see that the flare component is never softer than $\alpha=-0.2$. From Poutanen et al., in preparation.

flow is large enough $\left(R_{\mathrm{tr}} \gtrsim 100 R_{\mathrm{S}}\right)$ allowing the IR photons to escape (see Fig. 4). At the rising phase of the outburst, just a few day before the hard-to-soft spectral transition, a dip has been observed in the UV light curve of GX 339-4 Yan and Yu 2012). In Swift J1910.2-0546 a dip first appears in the IR, then optical and finally in the UV (N. Degenaar, priv. comm.). The timing of the dips is consistent with the collapse of the hot flow with increasing accretion rate (see Sect. 2.3).

It is worth noticing that the hard-to-soft and the soft-to-hard spectral transitions occur at different X-ray luminosities (e.g. Zdziarski et al. 2004). This hysteresis is most probably related to the fact that at the same luminosity the cold disc is further away 
from the central source on the rising phase of the outburst, than on the decline. The hysteresis should be then also reflected in the OIR spectra, namely the fast colour change should occur at a higher X-ray luminosity on the rising phase, than on the decline, as indeed observed.

Some BHBs, however, do show signatures of the jet emission in the OIR band. The most obvious examples is microquasar GRS 1915+105, whose radio light-curve was found to be very similar to the IR one with a few hours delay (Fender et al. 1997) favouring a common origin. It, however, accretes at a nearly Eddington rate and is hardly representative. The OIR spectrum of 4U 1543-47 and MAXI J1836-194 in the hard state is rather soft with $\alpha \sim-0.7$ (Kalemci et al. 2005; Russell et al. 2013), which is consistent with the optically thin synchrotron emission from the jet. Furthermore, the rms spectrum of the IR variability of XTE J1118+480 during the 2005 outburst is close to a power-law with $\alpha \sim-0.8$ (Hynes et al. 2006), implying probably the jet origin. And finally, GX 339-4 in the hard state demonstrated strong correlated IR and X-ray variability with the IR lagging by $0.1 \mathrm{~s}$, which could be interpreted as a signature of propagation delays between the X-ray producing accretion disc and the jet (Casella et al. 2010). It is well possible that three components (the irradiated disc, the hot flow and the jet) contribute to the OIR band and their contribution can vary not only from source to source, but also in the same source from the outburst to the outburst.

\subsection{X-ray variability}

In addition to the spectral properties, the variability in X-rays and longer wavelengths puts strong constraints on the models. In the hard state, the typical power-density spectra (PDS) that describe the X-ray variability can roughly be represented as a doubly-broken power-law with indices $0,-1$ and -2 from low to high frequencies. A more accurate description of the PDS is achieved by representing it with the Lorentzians (e.g. Nowak 2000; Axelsson et al. 2005). The main source of the short-term variability in $\mathrm{BHs}$ is believed to be fluctuations in the mass accretion rate, propagating through the accretion flow (Lvubarskii 1997).

Often QPOs are observed in the range $\sim 0.1-10 \mathrm{~Hz}$ (see reviews by Remillard and McClintock 2006; Done et al. 2007). Their frequencies show correlation with the X-ray flux, amplitude of Compton reflection and anti-correlation with the hardness ratio (see Figs 5 and 9). The origin of QPOs is often associated with the precession of orbits around the BH due to a misalignment of the $\mathrm{BH}$ and the orbital spins, known as Lense-Thirring precession (e.g., Stella and Vietri 1998), or with oscillation modes of the accretion flow itself (e.g., Wagoner et al. 2001). The problem with the Lense-Thirring precession models is that the frequency for test masses is a strong function of radius and the BH spin (see e.g. Schnittman et al. 2006). It is not clear why any specific radius gets selected to produce a QPO. If that radius is defined by the truncation of the cold disc, why the QPOs are then observed in the Comptonization spectrum? However, if the flow is hot and thick, it will precess as a solid body (Fragile et al. 2007) with the frequency mostly depending on its size (which is a function of the accretion rate) and weakly on the $\mathrm{BH}$ spin and the flow height-to-radius ratio. In such a case, the precession frequencies lie in the observed range and the model explains well their correlations with other quantities (Ingram et al. 2009; Ingram and Done 2011). 

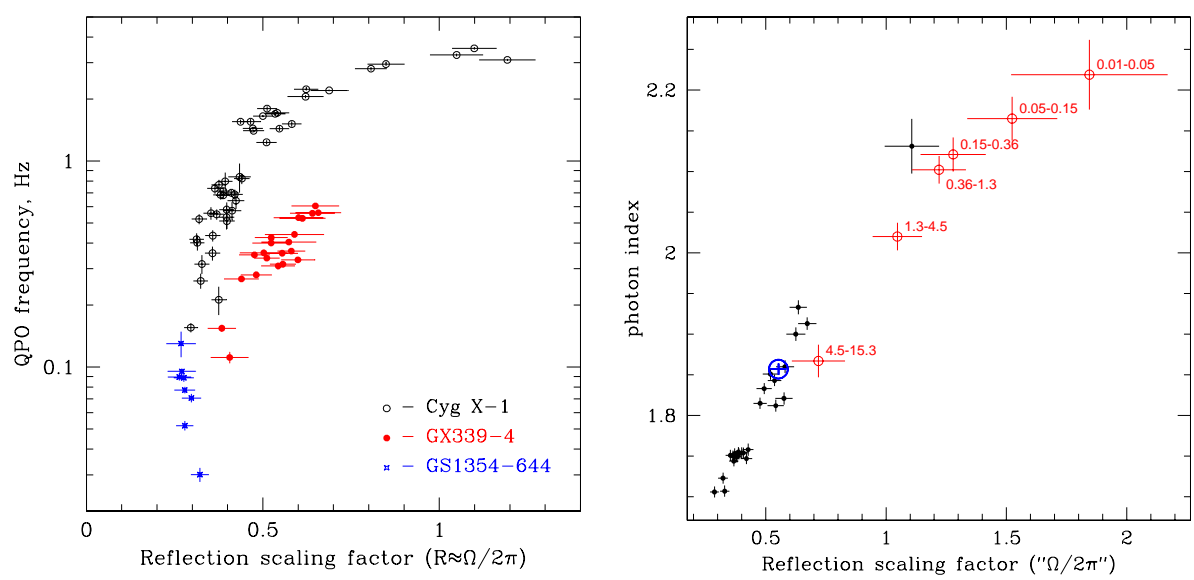

Fig. 9 Left: QPO frequency vs reflection correlation. From Gilfanov (2010). Right: Photon index - reflection correlation in the average spectra (same as Fig. 5 ) and in the Fourier frequency resolved spectra (FFRS) at different frequencies. From Gilfanov et al. (1999).

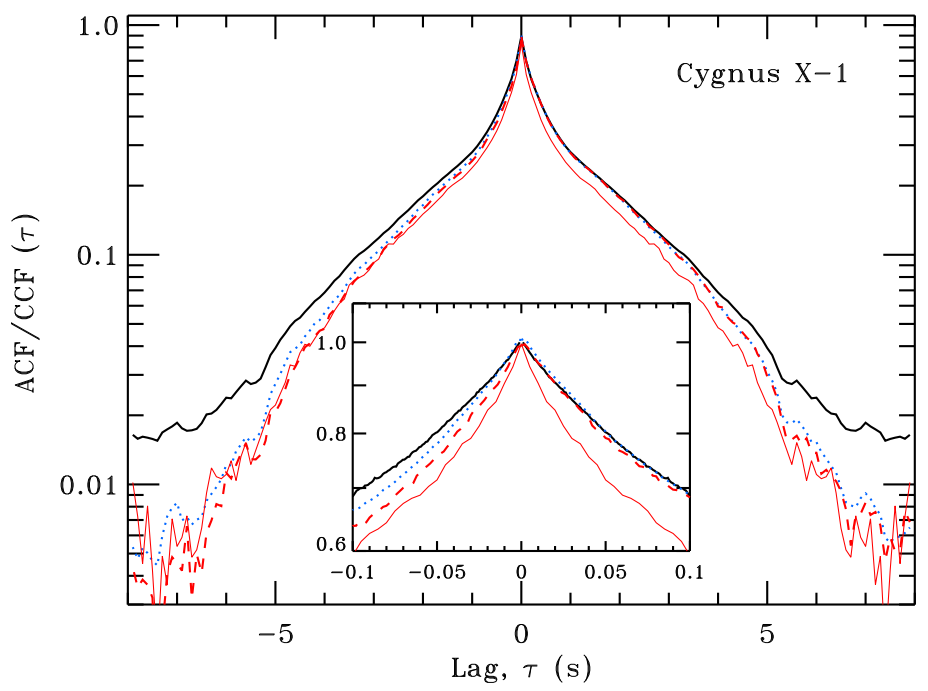

Fig. 10 The ACF and CCFs of Cygnus X-1 observed in December 1997. Solid curves show the ACFs for the 2-5 keV energy band (black solid curves) and the 24-40 keV band (red solid curves). The blue dotted curve shows the CCF between the 8-13 keV and the $2-5 \mathrm{keV}$ bands, and the red dashed curve represents the CCF for the $24-40 \mathrm{keV}$ vs the $2-5 \mathrm{keV}$ bands. The positive lag corresponds here to the hard photons lagging the soft ones. The CCFs are asymmetric, but the peak do not show any shift from zero lag. From Maccarone et al. (2000).

Another way of looking at the variability properties is through the autocorrelation function (ACF, which is related to the PDS via Fourier transform). The ACF becomes narrower at higher X-ray energies (Maccarone et al. 2000, see Fig. 10), which is equivalent to the excess variability at higher frequencies in the PDS at those energies. Moreover, the light curves at different energies are well correlated with each other, but the harder X-rays are delayed with respect to the soft X-rays (Miyamoto and Kitamoto 

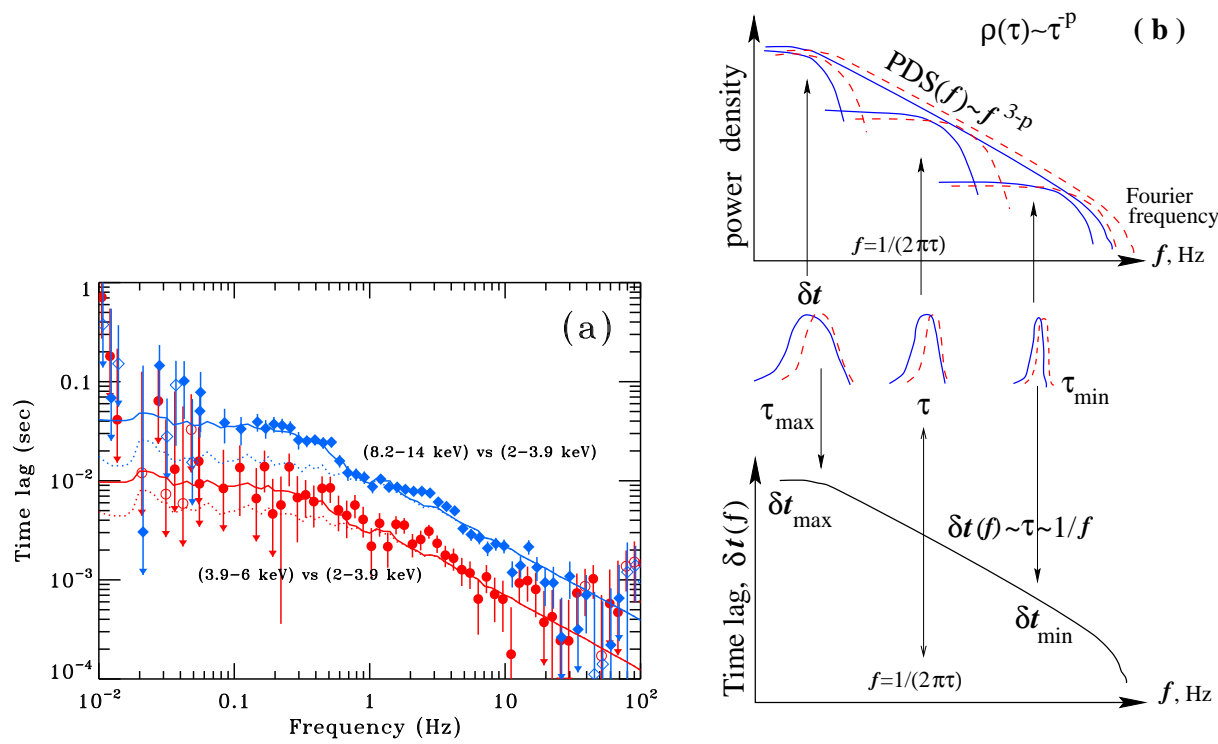

Fig. 11 (a): Time lags in Cyg X-1. The data points are from Nowak et al. (1999b). The dotted curves represent the model of Poutanen and Fabian (1999), where lags are produces by spectral evolution during flares. The solid curves have a contribution at low frequencies from the lags caused by reflection from the outer disc (Poutanen 2002). (b): A schematic picture how spectral evolution model produces time lags. The red dotted curves represent the flare light curve at high photon energies, while the blue solid curves represent those at low energies. Spectral hardening during flares produces hard time lags. Here $p$ is the index of the power-law probability distribution of flare duration $\tau$. From Poutanen (2001).

1989; Nowak et al. 1999b, 1999a, see Fig. 11). This effect is also reflected in the asymmetries of the cross-correlation function $(\mathrm{CCF})$ between the hard and the soft X-ray energy bands (Priedhorsky et al. 1979; Nolan et al. 1981; Maccarone et al. 2000, see Fig. (10).

In order to have a better understanding for these asymmetries, it is useful to look at the time lags $\Delta t$ between the light-curves at these X-ray energies as a function of the Fourier frequency $f$. For the hard state of Cygnus X-1 they are shown in Fig. 11]. As a function of energy $E$, the lags relative to energy $E_{0}$ follow the logarithmic law $\propto \ln \left(E / E_{0}\right)$. The rather large lags (exceeding $0.1 \mathrm{~s}$ ) were first interpreted as produced by Comptonization in a large Compton cloud (Kazanas et al. 1997). For harder photons more scatterings is required, thus they spend more time in the medium before escape and therefore are delayed. Such an interpretation not only causes a problem with the energetics of the cloud (requiring large energy release at distances $>10^{4} R_{\mathrm{S}}$ ), but also contradicts the energy dependence of the ACF width (Maccarone et al. 2000). On the other hand, the large lags, their frequency-dependence $f^{-1}$ and the logarithmic energy dependence can be naturally explained by spectral pivoting of a power-law-like spectrum if the characteristic time-scale of the evolution scales with the duration of shots $\tau$ dominating variability at frequency $f \approx 1 /(2 \pi \tau)$ (see Poutanen and Fabian 1999; Poutanen 2001; Kotov et al. 2001; Körding and Falcke 2004, and Fig. 11b). Among the first physical models explaining the spectral evolution was the flaring magnetic corona model of Poutanen and Fabian (1999). The observed linear relation between the flux and the rms (Uttley and McHardy 2001), however, argues against the indepen- 

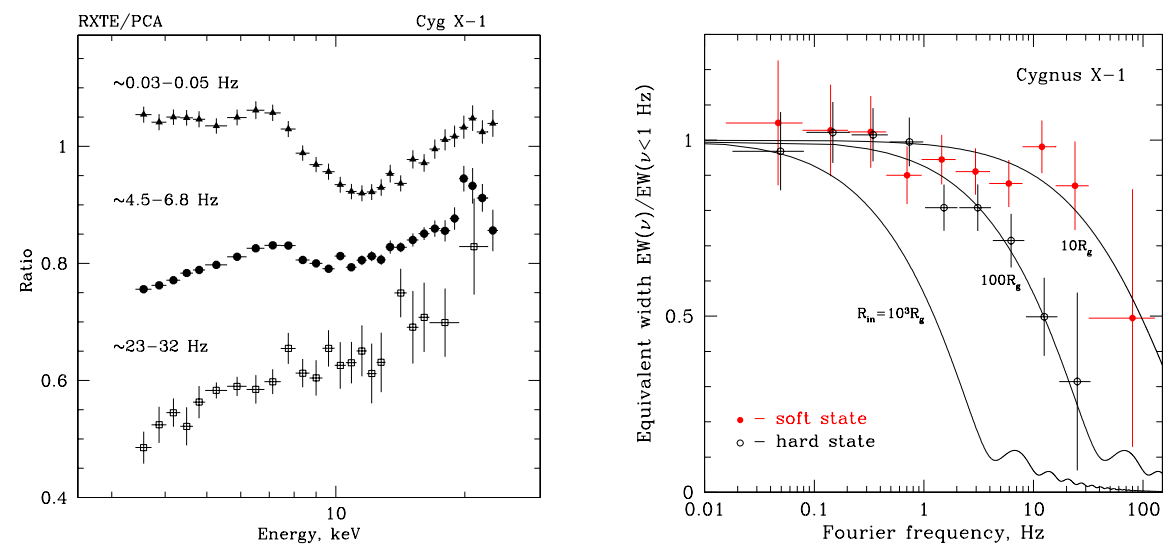

Fig. 12 Left: FFRS of Cyg X-1. From Revnivtsev et al. (1999). Right: equivalent width of the iron line as a function of Fourier frequency as measured from the FFRS. From Gilfanov et al. (2000).

dent shots (flares) as the source of variability. In the propagation model of Lyubarskii (1997), spectral evolution can arise when the accretion rate fluctuations propagate towards the BH into the zone with the harder spectra (Mivamoto and Kitamoto 1989; Kotov et al. 2001; Arévalo and Uttlev 2006) 3 This is consistent with the multi-zone hot flow model, because a lower flux of soft seed photon from the cold accretion disc as well as a stronger synchrotron self-absorption in the inner part of the flow produce harder Comptonization spectrum (Veledina et al. 2013a).

Additional contribution to the time lags are possible when the intrinsic X-rays from the hot flow are reflected from the distant matter, e.g. outer cold accretion disc (Kotov et al. 2001; Poutanen 2002). Due to the fluorescence and the energy dependence of the photo-electric opacity the contribution of reflection to the total spectrum is energy dependent. Thus the Fe line at $6.4 \mathrm{keV}$ and the Compton reflection bump above $10 \mathrm{keV}$ should show excess lags. Surprisingly, the deficit of lags (or "anti-lags") relative to the logarithmic dependence has been observed at these energies (Kotov et al. 2001). Clearly, this deficit cannot be caused by a simple reverberation and the light travel time effect. Instead it could be explained within the hot flow paradigm as follows. The outer parts of the flow which are close to the cold disc have softer spectra and larger reflection amplitude, while the inner flow produces harder spectrum with low or no reflection. Thus in the propagating fluctuation model, the reflection will be leading the hard spectrum causing negative delays at photon energies where it contributes Kotov et al. 2001). Delays due to reprocessing in the inner part of the cold disc are seen at high $f$ in GX 339-4 (Uttlev et al. 2011), while at low $f$ the disc photons lead the Comptonized photons, which is consistent with the propagation of fluctuations from the disc to the hot flow.

Further support to the truncated cold disc - hot inner flow scenario comes from the Fourier-frequency-resolved spectra (FFRS; Revnivtsev et al. 1999; Gilfanov et al. 2000), which are softer and have larger reflection amplitude at low Fourier frequencies (see Fig. 9 right and Fig. 12, left). This implies that soft X-rays are mostly produced

\footnotetext{
3 For the time lag production, this model is, however, mathematically identical to the flare evolution model.
} 
in the outer zones of the hot flow, closer to the cold reflecting medium, while the hard X-rays are produced in the inner zones that vary at high Fourier frequencies. The reduction of the equivalent width of the $6.4 \mathrm{keV}$ Fe line in the FFRS above $1 \mathrm{~Hz}$ (Fig. 12, right) suggests that the cold disc truncation radius in the hard state is about $100 R_{\mathrm{S}}$ (Revnivtsev et al. 1999; Gilfanov et al. 2000). Even larger truncation radius of the cold disc $\left(300-700 R_{\mathrm{S}}\right)$ was measured in the low-extinction BH transient XTE J1118+480 (Chaty et al. 2003; Yuan et al. 2005), where the peak is clearly seen in the UV. In the soft state instead the truncation radius is small $\left(<10 R_{\mathrm{S}}\right)$.

\subsection{Optical (IR and UV) variability and its relations to the X-rays}

Data on the fast variability are now available not only in the X-rays, but also at lower energies. The first simultaneous observations in the optical and X-rays were carried out already 30 years ago by Motch et al. (1983) for GX 339-4. Although no confident conclusion could be reached because of the short duration of observations, the optical/X-ray CCF revealed a complicated structure with a precognition dip (i.e. anti-correlation) at negative lags corresponding to optical leading the $\mathrm{X}$ rays and a peak (i.e. correlation) at positive lags (see Fig. 13b). Recently, similar CCFs were obtained from the much longer duration simultaneous observations in three BHBs: XTE J1118+480 (Kanbach et al. 2001; Hynes et al. 2003; Malzac et al. 2003), Swift J1753.5-0127 (Durant et al. 2008, 2009, 2011; Hvnes et al. 2009a) and GX 339-4 (Gandhi et al. 2008, 2010). These data provide an important information on the interrelation between various components and give clues to their physical origin.

The observed CCF shape cannot be explained by a simple reprocessing model Kanbach et al. 2001; Hynes et al. 2003). However, if the optical emission consists of two components, e.g., one coming from the non-thermal synchrotron in the hot flow and another from reprocessed X-ray emission, the complex shape can be reproduced (Veledina et al. 2011a, see Fig. 13b). Increase of the mass accretion rate obviously causes an increase in the X-ray luminosity, but at the same time the optical synchrotron from the hot flow may drop because of higher self-absorption. A higher accretion rate can also lead to a decrease of the truncation radius, collapse of the hot flow at large radii, and the suppression of the OIR emission. Both scenarios leads to anti-correlation between optical and X-rays and to the negative contribution to the CCF, with the shape resembling that of the X-ray ACF. On the other hand, the second, reprocessed component correlates with the X-rays, but is delayed and smeared, giving rise to a positive CCF peaking at positive lags (optical delay). The combined CCF has a complicated shape consistent with the data (Fig. 13b). The PDS of the optical in this model consists of three components: the synchrotron (which has nearly identical shape to the X-ray PDS), the irradiated disc (which has less power at high frequencies because of smearing) and the cross-term of variable sign. The total optical variability is strongly reduced at low frequencies where the synchrotron and the disc vary out of phase (Veledina et al. 2011a, see Fig. 13a). These PDS shapes are very similar to that observed in GX 339-4 (see fig. 9 in Gandhi et al. 2010).

Further clues on the origin of the optical emission come from the QPOs seen in the light curves of a number of low-mass BHBs (Motch et al. 1983, 1985; Imamura et al. 1990; Steiman-Cameron et al. 1997; Hvnes et al. 2003; Durant et al. 2009; Gandhi et al. 2010). The optical, UV and X-ray QPOs in XTE J1118+480 all have the same frequency, which evolves during the two months of observations (Hynes et al. 2003). There 

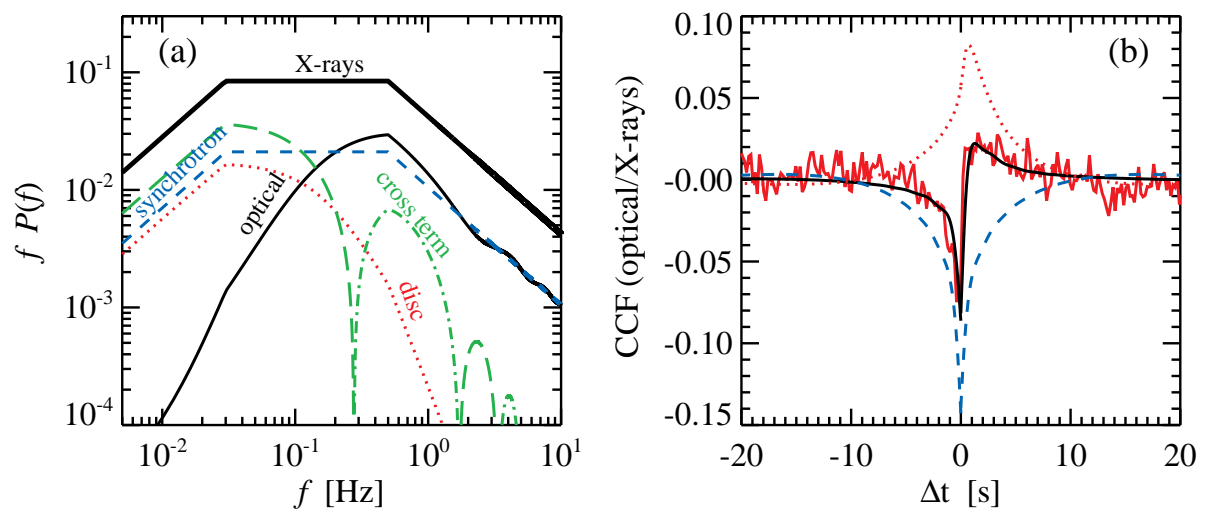

Fig. 13 Two-component model for the optical/X-ray CCF. (a) The PDSs of the X-ray (upper double-broken line) and optical light curves (thin solid line). Three terms contributing to the optical PDS are also shown: synchrotron (blue dashed), reprocessing in the disc (red dotted), and the cross term (green dot-dashed - positive contribution; long-dashed - negative contribution). (b) The optical/X-ray CCFs observed from Swift J1753.5-0127 in 2008 (red noisy curve, see fig. A3 in Durant et al. 2011). The model CCF is shown by the black solid curve, while contributions of the synchrotron and the reprocessed emission is shown by blue dashed and red dotted lines, respectively. Adapted from Veledina et al. (2011a).

is also a clear connection between the optical and X-ray light curves in the 2007 data on Swift J1753.5-0127 (see Durant et al. 2008; A. Veledina et al. in prep.) seen as a modulation at the optical QPO frequency in the optical/X-ray CCF. Similarly, GX 339-4 shows oscillations at the same frequency in the optical and X-rays (Motch et al. 1983; Gandhi et al. 2010, fig. 21). The question now arises how it is possible that the X-rays and OIR/UV vary at the same frequency and are phase-connected? This fact finds a simple explanation in the hybrid hot flow model because the hot flow can precess as a solid body (see Sect. 3.3) and therefore the long wavelength emission produced in the outer part of that flow is related to the X-rays produced in the inner part of the same flow (Veledina et al. 2013b).

Recently, periodic eclipses in the optical light curve of BHB Swift J1357.2-093313 were observed (Corral-Santana et al. 2013). If the period is related to the Keplerian frequency of the obscuring region, the sharpness of the eclipses implies that the size of the optical emission region is below $20000 \mathrm{~km}$ (i.e. $<700 R_{\mathrm{S}}$ for a $10 \mathrm{M} \odot \mathrm{BH}$ ). These constraints are easily satisfied in the hot flow scenario.

\subsection{Polarisation}

Polarisation degree and polarisation angle provide two more observational constraints on the emission models. The only indication of the X-ray polarisation from BHB goes back to the OSO-8 satellite (Weisskopf et al. 1977), which measured $3.1 \pm 1.7 \%$ linear polarisation from Cyg X-1 at $2.6 \mathrm{keV}$. Such a polarisation can be produced by Compton scattering if the geometry of the X-ray emitting region is a flattened disc-like structure ( $H / R \sim 0.2$ according to the calculations of Lightman and Shapiro 1976), consistent with the hot flow scenario. 
Recently, strong linear polarisation $(\Pi=67 \pm 30 \%)$ in the soft $\gamma$-rays above $400 \mathrm{keV}$ was detected in Cyg X-1 with the IBIS instrument onboard INTEGRAL Laurent et al. 2011). Similar polarisation $(\Pi=76 \pm 15 \%)$ was also observed with the SPI spectrometer (Jourdain et al. 2012b). The polarisation angle of $40^{\circ}-42^{\circ}$ is about $60^{\circ}$ away from the radio jet axis at $\approx-20^{\circ}$ (Jourdain et al. 2012b; Zdziarski et al. 2012). Such a large polarisation degree in the $\mathrm{MeV}$ range is extremely difficult to get in any scenario. Synchrotron jet emission from non-thermal electrons in a highly ordered magnetic field can have a large polarisation degree (up to $\sim 70$ per cent) in the optically thin part of the spectrum, and indeed a high polarisation in the radio and the optical bands reaching 30-50 per cent is observed from extragalactic relativistic jets (Impev et al. 1991; Wills et al. 1992; Lister 2001; Marscher et al. 2002; Ikejiri et al. 2011). However, this scenario also needs a very hard electron spectrum as well as an extreme fine-tuning to reproduce the spectral cutoff at a few $\mathrm{MeV}$ (Zdziarski et al. 2012). In the hot-flow scenario, the $\mathrm{MeV}$ photons are produced by non-thermal Compton scattering of the $100 \mathrm{keV}$ photons by electrons with Lorentz factors $\gamma \sim 2-4$. These electrons cannot be isotropic, because no significant polarisation is expected in that case (Poutanen 1994). This then implies that they must have nearly one-dimensional motion, e.g. along the large-scale magnetic field lines threading the flow. The $60^{\circ}$ offset of the polarisation vector relative to the jet axis then implies the inclined field lines. If the measured high polarisation degree is indeed real, this would put strong constraints on the physics of particle acceleration in the hot flow and the magnetic field geometry.

In the OIR bands, polarisation is very small and does not exceed a few per cent (Schultz et al. 2004; Shahbaz et al. 2008; Russell and Fender 2008; Chaty et al. 2011). In the hot-flow scenario, polarisation degree of the optically thin synchrotron radiation in the OIR band is expected to be essentially zero (independently of the magnetic field geometry) because the Faraday rotation angle exceeds $10^{5}$ rad (Veledina et al. 2013a). In the optically thick regime, the intrinsic polarisation (parallel to the magnetic field lines) is not more than about 10 per cent even for the ordered magnetic field (Pacholczyk and Swihart 1967; Ginzburg and Syrovatskii 1969). Faraday rotation in the disc atmosphere can still essentially depolarise that emission. The reprocessed emission from the outer disc can be slightly polarised because magnetic field there is smaller. If the jet were responsible for the OIR emission, one would expect instead a much higher polarisation because its radiation is optically thin, not consistent with the data. The observed small polarisation can also be produced by dust/electron scattering in the source vicinity or by the interstellar dust.

\section{Summary}

The purely thermal hot-flow model was shown to be consistent with many X-ray characteristics. However, that model fails to account for the $\mathrm{MeV}$ tails and a number of OIR properties. Addition of a small, energetically-negligible non-thermal component to the electron distribution dramatically changes the prediction of the model. The hybrid hot-flow model is now successful in explaining the following facts:

1. stability of spectra with photon index $\Gamma \sim 1.6-1.8$ and the cutoff at $\sim 100 \mathrm{keV}$ in the hard state (Poutanen and Vurm 2009; Malzac and Belmont 2009),

2. concave X-ray spectrum (Kotov et al. 2001; Veledina et al.|2013a),

3. low level of the X-ray and OIR polarisation (Veledina et al. 2013a), 
4. presence of the MeV tail in the hard state (Poutanen and Vurm 2009; Malzac and Belmont 2009),

5. softening of the X-ray spectrum with decreasing luminosity below $\sim 10^{-2} L_{\mathrm{Edd}}$ Veledina et al. 2011b),

6. weakness of the cold accretion disc component in the hard state,

7. correlation between the spectral index, the reflection amplitude, the width of the iron line and the frequency of the quasi-periodic oscillations,

8. hard X-ray lags with logarithmic energy dependence (Kotov et al.|2001),

9. non-thermal OIR excesses and flat OIR spectra (Veledina et al. 2013a),

10. OIR colours of the flares in the hard state (Poutanen et al., in prep.),

11. strong correlation between OIR and X-ray emission and a complicated CCF shape (Veledina et al. 2011a),

12. quasi-periodic oscillations at the same frequency in the X-ray and optical bands (Veledina et al. 2013b).

The model does not explain the radio points and the soft IR spectra. The jet is obviously a better model for those data. We, however, struggle to find any other observational fact that could be in conflict with the hybrid hot flow - truncated cold disc scenario.

Recently, the jet paradigm became popular and it was claimed that the jets are responsible not only for the radio emission from the BHBs, but also the OIR and even the $\mathrm{X}$-ray emission. Unfortunately, that model is in contradiction with dozens of observed facts (see Veledina et al. 2013a, and references therein), which are usually ignored by the model proponents. When new data appear, they often are rather puzzling and difficult to understand within the available paradigms. However, it would be beneficial for the community when introducing brand new models to check also whether those models satisfy other observational constraints.

In spite of a serious progress in understanding of the viscosity in accretion discs around $\mathrm{BHs}$, there are still many open questions. If non-thermal particles are present in the hot flow, it is now time to understand what is their nature. How are they accelerated: in shocks or in magnetic reconnections events, or maybe via diffusive acceleration by MHD turbulence? How are they related to the magneto-rotational instability that presumably drives the accretion? We hope that the observational advances will soon be reflected in the advance of the theory.

Acknowledgements The work was partially supported by the Academy of Finland grant 268740 (JP) and the Finnish Doctoral Program in Astronomy and Space Physics (AV). We thank Tomaso Belloni, Andrzej Zdziarski, and Feng Yuan for valuable comments.

\section{References}

V.A. Aref'ev, M.G. Revnivtsev, A.A. Lutovinov, R.A. Sunyaev, Astronomy Letters 30, 669674 (2004).

P. Arévalo, P. Uttley, MNRAS 367, 801-814 (2006).

M. Axelsson, L. Borgonovo, S. Larsson, A\&A 438, 999-1012 (2005).

R. Belmont, J. Malzac, A. Marcowith, A\&A 491, 617-631 (2008).

A.M. Beloborodov, in High Energy Processes in Accreting Black Holes, ed. by J. Poutanen, R. Svensson (ASP Conf. Ser., vol. 161, 1999a), pp. 295-314.

A.M. Beloborodov, ApJL 510, L123-L126 (1999b).

R.D. Blandford, A. Königl, ApJ 232, 34-48 (1979).

M.M. Buxton, C.D. Bailyn, ApJ 615, 880-886 (2004).

M.M. Buxton, C.D. Bailyn, H.L. Capelo, et al., AJ 143, 130 (2012). 
M. Cadolle Bel, M. Ribó, J. Rodriguez, et al., ApJ 659, 549-560 (2007).

M. Cadolle Bel, J. Rodriguez, P. D'Avanzo, et al., A\&A 534, 119 (2011).

M. Cadolle Bel, S. Corbel, A. Veledina, et al., in IAU Symposium, ed. by C.M. Zhang, T. Belloni, M. Méndez, S.N. Zhang (IAU Symp., vol. 290, 2013), pp. 17-20.

P. Casella, T.J. Maccarone, K. O'Brien, et al., MNRAS 404, 21-25 (2010).

A. Celotti, A.C. Fabian, M.J. Rees, MNRAS 255, 419-422 (1992).

S. Chaty, G. Dubus, A. Raichoor, A\&A 529, 3 (2011).

S. Chaty, C.A. Haswell, J. Malzac, et al. MNRAS 346, 689-703 (2003).

C.Y. Chiang, C. Done, M. Still, O. Godet, MNRAS 403, 1102-1112 (2010).

A. Constantin, P. Green, T. Aldcroft, et al., ApJ 705, 1336-1355 (2009).

P.S. Coppi, MNRAS 258, 657-683 (1992).

P.S. Coppi, in High Energy Processes in Accreting Black Holes, ed. by J. Poutanen, R. Svensson (ASP Conf. Ser., vol. 161, 1999), pp. 375-403.

S. Corbel, H. Aussel, J.W. Broderick, et al., MNRAS 431, 107-111 (2013).

J.M. Corral-Santana, J. Casares, T. Muñoz-Darias, et al., Sci 339, 1048-1051 (2013).

C. Cunningham, ApJ 208, 534-549 (1976)

S. Das, P.A. Becker, T. Le, ApJ 702, 649-659 (2009).

T. Dinçer, E. Kalemci, M.M. Buxton, et al., ApJ 753, 55 (2012).

J. Ding, F. Yuan, E. Liang, ApJ 708, 1545-1550 (2010).

C. Done, in Accretion Processes in Astrophysics, ed. by I. González Martínez-País, T. Shahbaz,

J. Casares Velázquez (Cambridge University Press, Cambridge, 2013), arXiv:1008.2287.

C. Done, M. Gierliński, A. Kubota, A\&ARv 15, 1-66 (2007).

R. Droulans, R. Belmont, J. Malzac, E. Jourdain, ApJ 717, 1022-1036 (2010).

M. Durant, P. Gandhi, T. Shahbaz, et al., ApJL 682, L45-L48 (2008).

M. Durant, P. Gandhi, T. Shahbaz, et al., MNRAS 392, 309-324 (2009).

M. Durant, T. Shahbaz, P. Gandhi, et al., MNRAS 410, 2329-2338 (2011).

A.A. Esin, J.E. McClintock, R. Narayan, ApJ 489, 865-889 (1997).

A.A. Esin, R. Narayan, W. Cui, et al., ApJ 505, 854-868 (1998).

A.A. Esin, J.E. McClintock, J.J. Drake, et al., ApJ 555, 483-488 (2001).

R.P. Fender, G.G. Pooley, C. Brocksopp, S.J. Newell, MNRAS 290, 65-69 (1997).

E.L. Fitzpatrick, PASP 111, 63-75 (1999).

P.C. Fragile, O.M. Blaes, ApJ 687, 757-766 (2008).

P.C. Fragile, O.M. Blaes, P. Anninos, J.D. Salmonson, ApJ 668, 417-429 (2007).

F. Frontera, E. Palazzi, A.A. Zdziarski, et al., ApJ 546, 1027-1037 (2001).

A.A. Galeev, R. Rosner, G.S. Vaiana, ApJ 229, 318-326 (1979).

E. Gallo, S. Migliari, S. Markoff, et al., ApJ 670, 600-609 (2007).

P. Gandhi, K. Makishima, M. Durant, et al., MNRAS 390, L29-L33 (2008).

P. Gandhi, V.S. Dhillon, M. Durant, et al., MNRAS 407, 2166-2192 (2010).

P. Gandhi, A.W. Blain, D.M. Russell, et al., ApJL 740, L13 (2011).

G. Ghisellini, P.W. Guilbert, R. Svensson, ApJL 334, L5-L8 (1988).

G. Ghisellini, F. Haardt, R. Svensson, MNRAS 297, 348-354 (1998).

M. Gierliński, C. Done, K. Page, MNRAS 392, 1106-1114 (2009).

M. Gierlinski, A.A. Zdziarski, C. Done, et al., MNRAS 288, 958-964 (1997)

M. Gierliński, A.A. Zdziarski, J. Poutanen, et al., MNRAS 309, 496-512 (1999).

M. Gilfanov, in The Jet Paradigm, ed. by T. Belloni (Lecture Notes in Physics, vol. 794, Berlin, 2010), pp. 17-51.

M. Gilfanov, E. Churazov, M. Revnivtsev, A\&A 352, 182-188 (1999).

M. Gilfanov, E. Churazov, M. Revnivtsev, MNRAS 316, 923-928 (2000).

V.L. Ginzburg, S.I. Syrovatskii, ARA\&A 7, 375-420 (1969).

J.E. Grove, W.N. Johnson, R.A. Kroeger, et al., ApJ 500, 899-908 (1998).

M. Gu, X. Cao, MNRAS 399, 349-356 (2009).

F. Haardt, L. Maraschi, ApJ 413, 507-517 (1993).

F. Haardt, L. Maraschi, G. Ghisellini, ApJL 432, L95-L99 (1994).

K.B. Henisey, O.M. Blaes, P.C. Fragile, ApJ 761, 18 (2012).

M. Hoshino, ApJ 773, 118 (2013).

R.I. Hynes, K. O'Brien, K. Horne, et al., MNRAS 299, 37-41 (1998).

R.I. Hynes, C.W. Mauche, C.A. Haswell, et al., ApJL 539, L37-L40 (2000),

R.I. Hynes, C.A. Haswell, S. Chaty, et al., MNRAS 331, 169-179 (2002).

R.I. Hynes, C.A. Haswell, W. Cui, et al., MNRAS 345, 292-310 (2003).

R.I. Hynes, E.L. Robinson, K.J. Pearson, et al., ApJ 651, 401-407 (2006). 
R.I. Hynes, K. O'Brien, F. Mullally, T. Ashcraft, MNRAS 399, 281-286 (2009a).

R.I. Hynes, C.K. Bradley, M. Rupen, et al., MNRAS 399, 2239-2248 (2009b).

A. Ibragimov, J. Poutanen, M. Gilfanov, et al., MNRAS 362, 1435-1450 (2005).

S. Ichimaru, ApJ 214, 840-855 (1977).

Y. Ikejiri, M. Uemura, M. Sasada, et al., PASJ 63, 639-675 (2011).

J.N. Imamura, J. Kristian, J. Middleditch, T.Y. Steiman-Cameron, ApJ 365, 312-316 (1990).

C.D. Impey, C.R. Lawrence, S. Tapia, ApJ 375, 46-68 (1991).

A. Ingram, C. Done, MNRAS 415, 2323-2335 (2011).

A. Ingram, C. Done, P.C. Fragile, MNRAS 397, 101-105 (2009).

R.K. Jain, C.D. Bailyn, J.A. Orosz, et al., ApJL 554, L181-L184 (2001).

E. Jourdain, J.P. Roques, J. Malzac, ApJ 744, 64 (2012a).

E. Jourdain, J.P. Roques, M. Chauvin, D.J. Clark, ApJ 761, 27 (2012b).

E. Kalemci, J.A. Tomsick, M.M. Buxton, et al., ApJ 622, 508-519 (2005).

E. Kalemci, T. Dincer, J.A. Tomsick, et al. ApJ, in press, arXiv:1310.5482 (2013).

G. Kanbach, C. Straubmeier, H.C. Spruit, T. Belloni, Nature 414, 180-182 (2001).

D. Kazanas, X.-M. Hua, L. Titarchuk, ApJ 480, 735-740 (1997).

M. Kolehmainen, C. Done, M. Díaz Trigo, MNRAS, in press, arXiv:1310.1219 (2013).

E. Körding, H. Falcke, A\&A 414, 795-806 (2004).

O. Kotov, E. Churazov, M. Gilfanov, MNRAS 327, 799-807 (2001).

J.H. Krolik, ApJL 498, L13-L16 (1998).

P. Laurent, J. Rodriguez, J. Wilms, et al., Sci 332, 438 (2011).

A.P. Lightman, S.L. Shapiro, ApJ 203, 701-703 (1976).

J.C. Ling, W.A. Wheaton, P. Wallyn, et al., ApJ 484, 375-382 (1997).

M.L. Lister, ApJ 562, 208-232 (2001).

Y.E. Lyubarskii, MNRAS 292, 679-685 (1997).

T.J. Maccarone, MNRAS 360, 68-72 (2005).

T.J. Maccarone, P.S. Coppi, J. Poutanen, ApJL 537, L107-L110 (2000).

R. Mahadevan, Nature 394, 651-653 (1998).

K. Makishima, H. Takahashi, S. Yamada, et al. PASJ 60, 585-604 (2008).

J. Malzac, R. Belmont, MNRAS 392, 570-589 (2009).

J. Malzac, R. Belmont, A.C. Fabian, MNRAS 400, 1512-1520 (2009).

J. Malzac, A.M. Beloborodov, J. Poutanen, MNRAS 326, 417-427 (2001).

J. Malzac, T. Belloni, H.C. Spruit, G. Kanbach, A\&A 407, 335-345 (2003).

A.P. Marscher, ApJ 216, 244-256 (1977).

A.P. Marscher, S.G. Jorstad, J.R. Mattox, A.E. Wehrle, ApJ 577, 85-97 (2002).

J.E. McClintock, R. Narayan, J.F. Steiner, Space Sci. Rev., in press, arXiv:1303.1583 (2013).

J.E. McClintock, C.A. Haswell, M.R. Garcia, et al., ApJ 555, 477-482 (2001).

M.L. McConnell, A.A. Zdziarski, K. Bennett, et al., ApJ 572, 984-995 (2002).

M. McConnell, D. Forrest, J. Ryan, et al., ApJ 424, 933-939 (1994).

S. Miyamoto, S. Kitamoto, Nature 342, 773 (1989).

C. Motch, M.J. Ricketts, C.G. Page, et al., A\&A 119, 171-176 (1983).

C. Motch, S.A. Ilovaisky, C. Chevalier, P. Angebault, Space Sci. Rev. 40, 219-224 (1985).

M.P. Muno, J. Mauerhan, ApJL 648, L135-L138 (2006).

R. Narayan, I. Yi, ApJL 428, L13-L16 (1994).

R. Narayan, I. Yi, ApJ 452, 710-735 (1995).

R. Narayan, R. Mahadevan, E. Quataert, in Theory of Black Hole Accretion Disks, ed. by M.A. Abramowicz, G. Bjornsson, J.E. Pringle (Cambridge University Press, Cambridge, 1998), pp. 148-182.

S. Nayakshin, F. Melia, ApJS 114, 269-288 (1998).

A. Niedźwiecki, F.-G. Xie, A.A. Zdziarski, MNRAS 420, 1195-1206 (2012).

P.L. Nolan, D.E. Gruber, J.L. Matteson, et al., ApJ 246, 494-501 (1981).

D.I. Novikov, K.S. Thorne, in Black Holes (Les Astres Occlus), ed. by C. Dewitt, B.S. Dewitt (Gordon \& Breach, New York, 1973), pp. 343-450.

M.A. Nowak, MNRAS 318, 361-367 (2000).

M.A. Nowak, J. Wilms, J.B. Dove, ApJ 517, 355-366 (1999a).

M.A. Nowak, B.A. Vaughan, J. Wilms, et al., ApJ 510, 874-891 (1999b)

K. O'Brien, K. Horne, R.I. Hynes, et al., MNRAS 334, 426-434 (2002).

J.A. Orosz, J.F. Steiner, J.E. McClintock, et al., ApJ 730, 75 (2011).

F. Özel, D. Psaltis, R. Narayan, ApJ 541, 234-249 (2000).

A.G. Pacholczyk, T.L. Swihart, ApJ 150, 647-650 (1967). 
J. Poutanen, ApJS 92, 607-609 (1994).

J. Poutanen, in Theory of Black Hole Accretion Disks, ed. by M.A. Abramowicz, G. Björnsson,

J.E. Pringle (Cambridge University Press, Cambridge, 1998), pp. 100-122.

J. Poutanen, Adv. Sp. Res. 28, 267-280 (2001).

J. Poutanen, MNRAS 332, 257-270 (2002).

J. Poutanen, P.S. Coppi, Physica Scripta T 77, 57-60 (1998).

J. Poutanen, A.C. Fabian, MNRAS 306, L31-L37 (1999).

J. Poutanen, R. Svensson, ApJ 470, 249-268 (1996).

J. Poutanen, I. Vurm, ApJL 690, L97-L100 (2009).

J. Poutanen, A.A. Zdziarski, in New Views on Microquasars, ed. by P. Durouchoux, Y. Fuchs,

J. Rodriguez (Center for Space Physics, Kolkata, 2003), pp. 95-100.

J. Poutanen, J.H. Krolik, F. Ryde, MNRAS 292, L21-L25 (1997).

W. Priedhorsky, G.P. Garmire, R. Rothschild, et al., ApJ 233, 350-363 (1979).

F. Rahoui, M. Coriat, S. Corbel, et al., MNRAS 422, 2202-2212 (2012).

R.A. Remillard, J.E. McClintock, ARA\&A 44, 49-92 (2006).

M. Revnivtsev, M. Gilfanov, E. Churazov, A\&A 347, 23-26 (1999).

M. Revnivtsev, M. Gilfanov, E. Churazov, A\&A 380, 520-525 (2001).

M.A. Riquelme, E. Quataert, P. Sharma, A. Spitkovsky, ApJ 755, 50 (2012).

D.M. Russell, R.P. Fender, MNRAS 387, 713-723 (2008).

D.M. Russell, D. Maitra, R.J.H. Dunn, S. Markoff, MNRAS 405, 1759-1769 (2010).

D.M. Russell, D. Maitra, R.J.H. Dunn, R.P. Fender, MNRAS 416, 2311-2317 (2011).

D.M. Russell, P.A. Curran, T. Muñoz-Darias, et al., MNRAS 419, 1740-1751 (2012).

D.M. Russell, T.D. Russell, J.C.A. Miller-Jones, et al., ApJL 768, L35 (2013)

J.D. Schnittman, J. Homan, J.M. Miller, ApJ 642, 420-426 (2006).

J. Schultz, P. Hakala, J. Huovelin, Balt. Astr. 13, 581-595 (2004).

T. Shahbaz, R.P. Fender, C.A. Watson, K. O'Brien, ApJ 672, 510-515 (2008).

N.I. Shakura, R.A. Sunyaev, A\&A 24, 337-355 (1973).

S.L. Shapiro, A.P. Lightman, D.M. Eardley, ApJ 204, 187-199 (1976).

M. Shidatsu, Y. Ueda, F. Tazaki, et al., PASJ 63, 785 (2011).

M.A. Sobolewska, I.E. Papadakis, C. Done, J. Malzac, MNRAS 417, 280-288 (2011).

T.Y. Steiman-Cameron, J.D. Scargle, J.N. Imamura, J. Middleditch, ApJ 487, 396-401 (1997). L. Stella, M. Vietri, ApJL 492, L59-L62 (1998).

B.E. Stern, M.C. Begelman, M. Sikora, R. Svensson, MNRAS 272, 291-307 (1995a).

B.E. Stern, J. Poutanen, R. Svensson, et al., ApJL 449, L13-L17 (1995b).

P. Uttley, I.M. McHardy, MNRAS 323, L26-L30 (2001).

P. Uttley, T. Wilkinson, P. Cassatella, et al., MNRAS 414, 60-64 (2011).

A. Veledina, J. Poutanen, A. Ingram, ApJ, 778, 165 (2013b).

A. Veledina, J. Poutanen, I. Vurm, ApJL 737, L17 (2011a).

A. Veledina, J. Poutanen, I. Vurm, MNRAS 430, 3196-3212 (2013a).

A. Veledina, I. Vurm, J. Poutanen, MNRAS 414, 3330-3343 (2011b).

I. Vurm, J. Poutanen, ApJ 698, 293-316 (2009).

R.V. Wagoner, A.S. Silbergleit, M. Ortega-Rodríguez, ApJL 559, L25-L28 (2001).

G. Wardziński, A.A. Zdziarski, MNRAS 325, 963-971 (2001).

M.C. Weisskopf, E.H. Silver, H.L. Kestenbaum, et al., ApJL 215, L65-L68 (1977).

B.J. Wills, D. Wills, N.J. Evans II, et al., ApJ 400, 96-114 (1992).

Q. Wu, M. Gu, ApJ 682, 212-217 (2008).

F.-G. Xie, A. Niedźwiecki, A.A. Zdziarski, F. Yuan, MNRAS 403, 170-178 (2010).

S. Yamada, K. Makishima, C. Done, et al., PASJ 65, 80 (2013).

Z. Yan, W. Yu, MNRAS 427, 11-15 (2012).

F. Yuan, R. Narayan, ARA\&A, submitted (2014).

F. Yuan, A.A. Zdziarski, MNRAS 354, 953-960 (2004).

F. Yuan, W. Cui, R. Narayan, ApJ 620, 905-914 (2005).

F. Yuan, E. Quataert, R. Narayan, ApJ 598, 301-312 (2003).

F. Yuan, A.A. Zdziarski, Y. Xue, X.-B. Wu, ApJ 659, 541-548 (2007).

A.A. Zdziarski, M. Gierliński, Progr. Theor. Phys. Suppl. 155, 99-119 (2004).

A.A. Zdziarski, P. Lubiński, M. Sikora, MNRAS 423, 663-675 (2012).

A.A. Zdziarski, P. Lubinski, D.A. Smith, MNRAS 303, L11-L15 (1999).

A.A. Zdziarski, W.N. Johnson, J. Poutanen, et al., in ESA SP-382: The Transparent Universe, ed. by C. Winkler, T.J.-L. Courvoisier, P. Durouchoux (ESA, Noordwijk, 1997), pp. 373380 . 
A.A. Zdziarski, J. Poutanen, J. Mikolajewska, et al., MNRAS 301, 435-450 (1998).

A.A. Zdziarski, J.E. Grove, J. Poutanen, A.R. Rao, S.V. Vadawale, ApJL 554, L45-L48 (2001).

A.A. Zdziarski, P. Lubiński, M. Gilfanov, M. Revnivtsev, MNRAS 342, 355-372 (2003).

A.A. Zdziarski, M. Gierliński, J. Mikołajewska, et al., MNRAS 351, 791-807 (2004). 